Provided for non-commercial research and education use. Not for reproduction, distribution or commercial use.

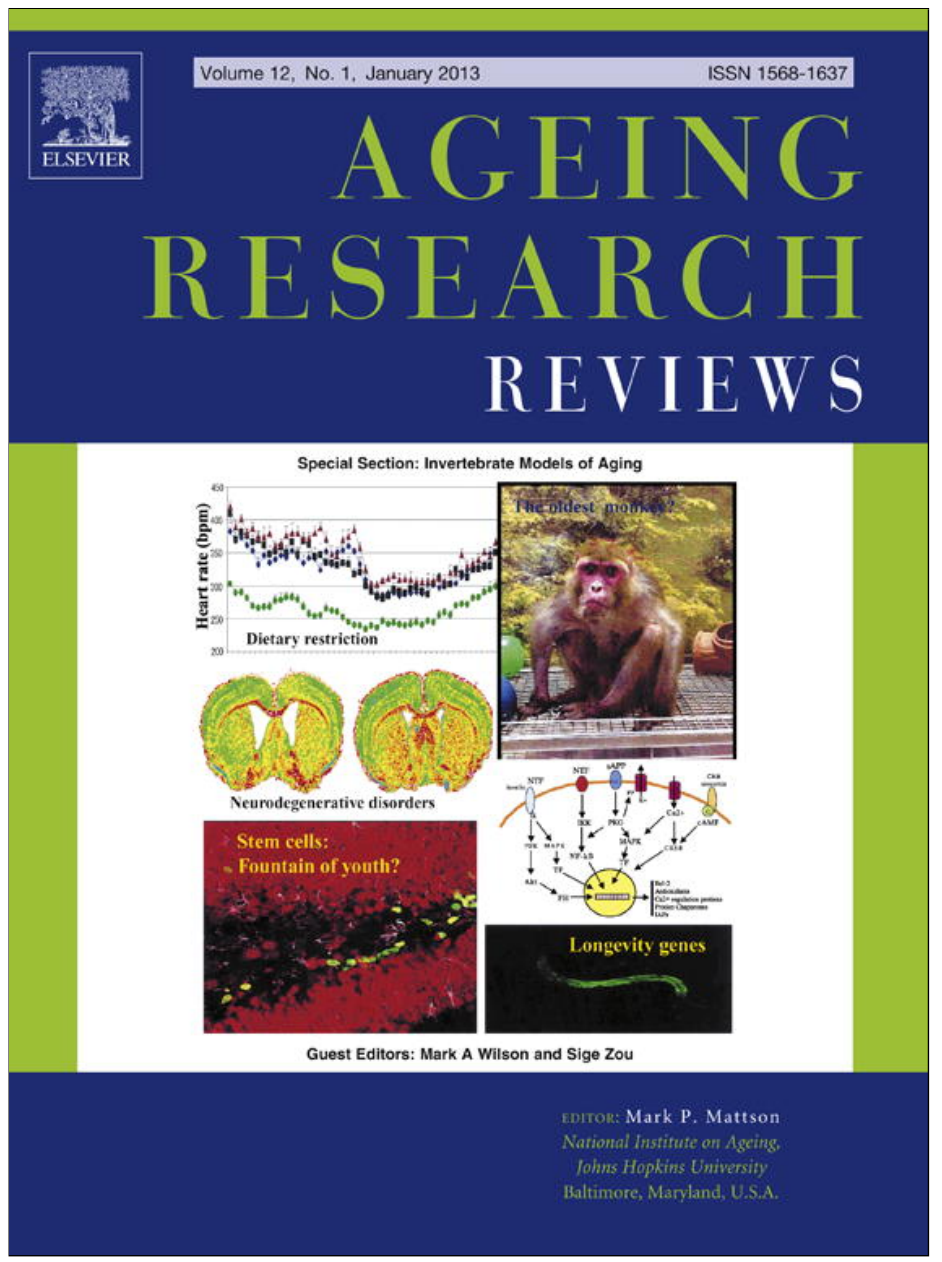

This article appeared in a journal published by Elsevier. The attached copy is furnished to the author for internal non-commercial research and education use, including for instruction at the authors institution and sharing with colleagues.

Other uses, including reproduction and distribution, or selling or licensing copies, or posting to personal, institutional or third party websites are prohibited.

In most cases authors are permitted to post their version of the article (e.g. in Word or Tex form) to their personal website or institutional repository. Authors requiring further information regarding Elsevier's archiving and manuscript policies are encouraged to visit:

http://www.elsevier.com/copyright 
Review

\title{
Autophagy and ageing: Insights from invertebrate model organisms
}

\author{
Eirini Lionaki ${ }^{1}$, Maria Markaki ${ }^{1}$, Nektarios Tavernarakis* \\ Institute of Molecular Biology and Biotechnology, Foundation for Research and Technology-Hellas, Heraklion 71110, Crete, Greece
}

\section{A R T I C L E I N F O}

Article history:

Received 15 December 2011

Received in revised form 3 May 2012

Accepted 8 May 2012

Available online 24 May 2012

\section{Keywords:}

Caenorhabditis elegans

Drosophila

Homeostasis

Insulin/IGF-1 signalling

Senescence

Stress

\begin{abstract}
A B S T R A C T
Ageing in diverse species ranging from yeast to humans is associated with the gradual, lifelong accumulation of molecular and cellular damage. Autophagy, a conserved lysosomal, self-destructive process involved in protein and organelle degradation, plays an essential role in both cellular and whole-animal homeostasis. Accumulating evidence now indicates that autophagic degradation declines with age and this gradual reduction of autophagy might have a causative role in the functional deterioration of biological systems during ageing. Indeed, loss of autophagy gene function significantly influences longevity. Moreover, genetic or pharmacological manipulations that extend lifespan in model organisms often activate autophagy. Interestingly, conserved signalling pathways and environmental factors that regulate ageing, such as the insulin/IGF-1 signalling pathway and oxidative stress response pathways converge on autophagy. In this article, we survey recent findings in invertebrates that contribute to advance our understanding of the molecular links between autophagy and the regulation of ageing. In addition, we consider related mechanisms in other organisms and discuss their similarities and idiosyncratic features in a comparative manner.
\end{abstract}

(c) 2012 Elsevier B.V. All rights reserved.

\section{Introduction}

Ageing is a complex process characterized by the progressive accumulation of damage to molecules, cells, tissues and organs that eventually leads to overall functional decline and increased vulnerability to disease and death. Although, stochastic and environmental factors undoubtedly contribute to the ageing process, intrinsic genetic determinants also modulate both lifespan and healthspan. During the past two decades, many studies in simple model organisms such as Saccharomyces cerevisiae, Caenorhabditis elegans and Drosophila melanogaster have culminated in the delineation of several signalling pathways that influence ageing (Bishop and Guarente, 2007a; Giannakou and Partridge, 2007; Guarente and Kenyon, 2000; Kenyon, 2010). Numerous genetic manipulations and treatments that influence the lifespan of diverse organisms interface with metabolism, nutrient sensing and stress response pathways.

Macroautophagy (hereafter, referred to as autophagy) is an evolutionarily conserved self-eating process by which cytoplasmic components including macromolecules and organelles are sequestered into double-membrane vesicles, the autophagosomes, and then delivered to lysosomes for degradation (Fig. 1; Yang and Klionsky, 2010). Autophagy-related genes (ATG) are highly

\footnotetext{
* Corresponding author. Tel.: +30 2810 391066; fax: +30 2810391067

E-mail address: tavernarakis@imbb.forth.gr (N. Tavernarakis).

1 These authors contributed equally.
}

conserved among eukaryotes. Functional analyses in invertebrate and mammalian models have revealed multiple roles for autophagy in various physiological contexts. A basal level of constitutive autophagy is crucial for routine clearance of the cytosol under normal conditions. Basal autophagy is critical for protein and organelle homeostasis and quality control in post-mitotic differentiated cells such as neurons. In addition, autophagy becomes activated in response to low nutrient availability, providing a source of nutrients and energy. Autophagy is also triggered as an adaptive response to a broad range of other extracellular or intracellular stressors such as hypoxia, heat, reactive oxygen species (ROS) and accumulation of damaged cytoplasmic components (Levine and Klionsky, 2004). Suppression of autophagy by knockout or knockdown of essential autophagy genes triggers apoptosis or necrosis in cells that would otherwise survive under stress conditions (reviewed in Kourtis and Tavernarakis, 2009; Mathew et al., 2009).

Autophagy may proceed as a non-selective catabolic process for bulk segregation and digestion of portions of the cytoplasm in the lysosome, but also, in certain cases, it can selectively target proteins and organelles such as mitochondria (mitophagy), ribosomes (ribophagy), peroxisomes (pexophagy), and endoplasmic reticulum (ER; reticulophagy), thus contributing to their turnover (reviewed in He and Klionsky, 2009; Yang and Klionsky, 2010). Autophagy appears to serve primarily a cytoprotective function by maintaining nutrient and energy homeostasis during starvation or by degrading damaged cellular components and invasive pathogens. Paradoxically, although autophagy is a 


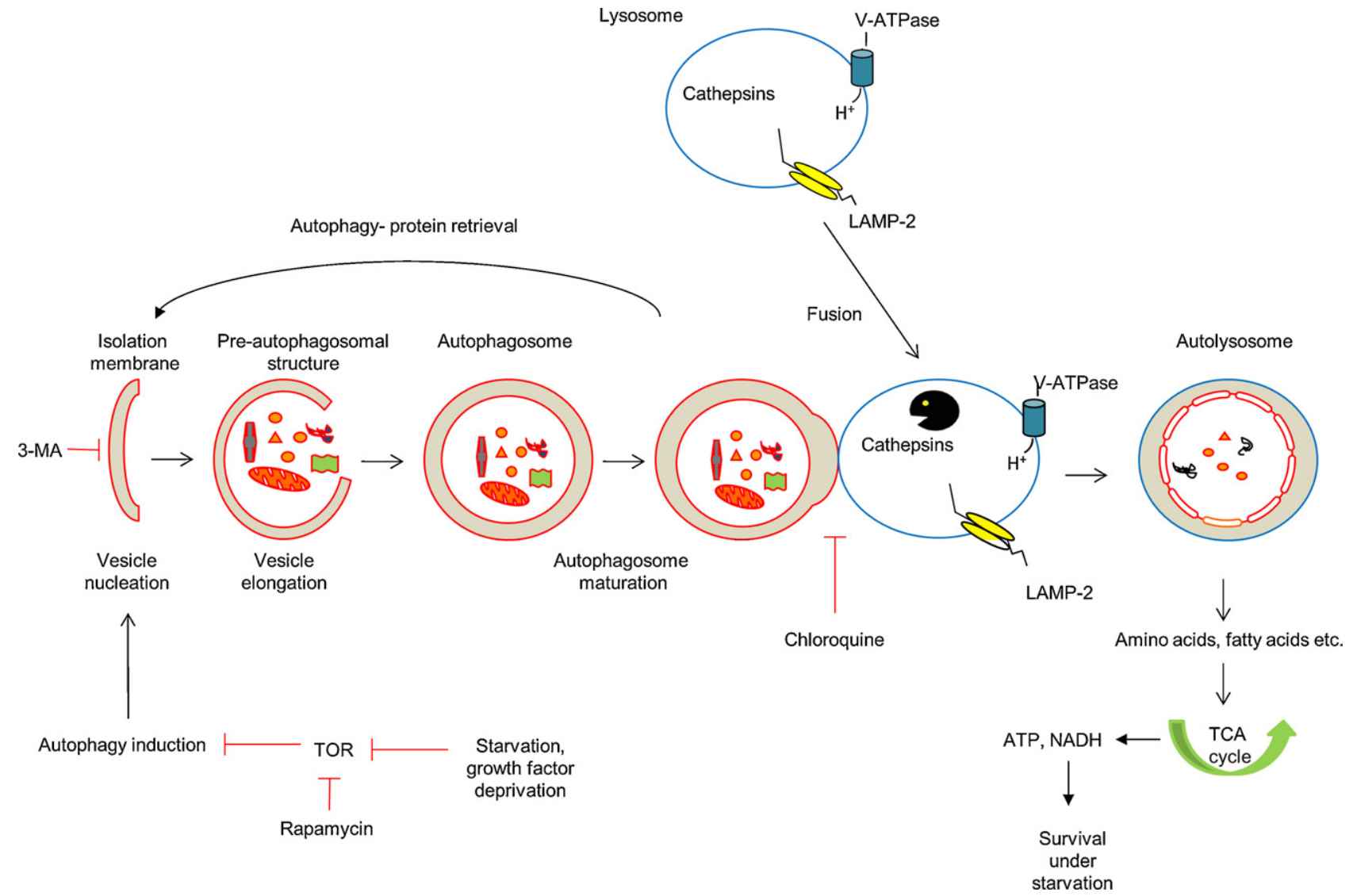

Fig. 1. The process of macroautophagy. Autophagy begins with the formation of the isolation membrane. A series of autophagy protein complexes coordinate the formation of double-membrane vesicles called autophagosomes. Autophagosomes can engulf bulk portions of cytoplasm non-specifically, including entire organelles, or target cargos specifically. The outer membrane of the autophagosome subsequently fuses with a lysosome generating an autolysosome, in which proteases, lipases, nucleases, and glycosidases degrade cytoplasmic material. Lysosomal permeases release the breakdown products into the cytosol for reutilization. Free amino acids and fatty acids are used as building blocks for new protein synthesis and ATP production, promoting homeostasis and survival at both the cell and whole organism level. Certain autophagy proteins are retrieved from autophagosomes for later reuse. Starvation or growth factor deprivation triggers autophagosome nucleation and elongation through TOR signalling. Pharmacological agents such as rapamycin can inhibit distinct steps of the autophagy pathway (red blocks). TOR, target of rapamycin; TCA, tricarboxylic acid; NADH, reduced form of nicotinamide adenine dinucleotide; 3-MA, 3-methyladenine; V-ATPase, vacuolar $\mathrm{H}^{+}$-ATPase; LAMP-2, lysosome-associated membrane protein 2.

predominantly homeostatic mechanism, it can also play a role in cell death, which is not restricted to developmental programmed cell death but extends to cell death that occurs in many pathological conditions. Excessive autophagy induced by extreme conditions, such as toxins and necrosis-triggering insults, might cause uncontrollable degradation or sequestration of cells contents into autophagosomes resulting in undesirable cell death if not properly regulated (reviewed in Kourtis and Tavernarakis, 2009; Samara and Tavernarakis, 2008; Yang and Klionsky, 2010).

Beyond its functions at the cellular level, autophagy has also been implicated in the regulation of whole organism healthspan and lifespan. Accumulating findings indicate that clearing cellular damage by autophagy is a common denominator of different lifespan - influencing pathways in diverse organisms including yeast, worms, flies and mammals (Bjedov et al., 2010; Giannakou and Partridge, 2007; Hansen et al., 2008; Hars et al., 2007; Levine and Kroemer, 2008; Toth et al., 2008). Here, we review recent research developments that highlight the interaction of autophagy with several evolutionarily conserved mechanisms linked to longevity. We focus on multicellular invertebrate model organisms such as C. elegans and D. rosophila, which have contributed important insights into the mechanisms of ageing. Further, we discuss the role of autophagy as an adaptive mechanism by which an organism responds to environmental fluctuations to preserve homeostasis and maintain functionality during ageing.

\section{The process of autophagy}

Although autophagy was initially identified in mammals, genetic studies in yeast provided fundamental insights into the molecular machinery involved in autophagic degradation (Huang and Klionsky, 2002). These studies identified many ATG that encode proteins involved in the induction of autophagy, the formation, expansion and maturation of autophagosomes and in the retrieval of autophagic proteins from mature autophagosomes (Klionsky, 2005; Klionsky et al., 2003). Conventional autophagy components include the Ulk1 complex (Atg1 in yeast, UNC-51 in worms and ULK1 in mammals), the class III phosphatidylinositol 3-kinase (PtdIns3K) complex, the Atg9 system, and two Atg7-mediated ubiquitin-like protein complexes (Atg8 and Atg12, conjugated with phosphatidylethanolamine and Atg5 respectively; the Atg12-Atg5 conjugate further interacts with Atg16; Xie and Klionsky, 2007). The first crucial event in the autophagic process is the induction or nucleation of the phagophore (also called the isolation membrane). A protein complex containing the Ser/Thr kinase Atg1 and two scaffolding proteins Atg13 and Atg17 is an essential positive regulator of autophagosome formation. Orthologues of these genes have been described in diverse species including $C$. elegans and Drosophila.

Several proteins involved in autophagy have been identified in C. elegans, including the Atg1 homolog UNC-51 (uncoordinated-51), 
the Atg13 homolog EPG-1 (ectopic PGL granules), and others (Melendez and Levine, 2009; Tian et al., 2009, 2010). In addition to its involvement in autophagy, Atg1/UNC-51 has a crucial role as a neuronal specific protein that functions in axonal elongation and guidance (Melendez and Levine, 2009). Autophagosome nucleation requires a complex containing Atg6 (bec-1 in C. elegans) that recruits the class III phosphatidylinositol 3-kinase VPS34 to generate phosphatidylinositol 3-phosphate. Expansion of autophagosome membranes involves two ubiquitin-like molecules, Atg12 and Atg8 (lgg-1 in C. elegans), an E1 ubiquitin activating enzyme (Atg7), two analogues of ubiquitin-conjugated enzymes (Atg10 and Atg3), an Atg8 modifying protease (Atg4), the protein target of Atg12 attachment (Atg5) and Atg16. In the first ubiquitin reaction, the E1-like Atg7 and the E2-like Atg10 covalently link Atg12 to Atg5. This Atg5-Atg12 conjugate subsequently interacts with Atg16 to form pre-autophagosomal structures. In the second ubiquitin reaction, Atg8 (LGG-1) is cleaved by the protease Atg4 and is conjugated to phosphatidylethanolamine (PE) by Atg7 and Atg3 (E2-like). This lipidated Atg8 (LGG-1) associates with newly forming autophagosome membranes. Upon completion of autophagosome formation, the Atg12-Atg5-Atg16 complex is released into the cytosol, whereas Atg8-PE stably associates with the autophagosomal membranes. This highly specific association has facilitated assays that exploit Atg8 localization to autophagosomes as a proxy to monitor autophagy in vivo (Chen and Klionsky, 2011; Rabinowitz and White, 2010). Lysosome docking and fusion occurs when the outer autophagosomal membrane fuses with the lysosomal membrane producing an autolysosome (an autophagic body in yeast). The remaining single-membrane that envelops the cargo is lysed and the population of Atg8-PE together with the enclosed cargo are released into the lysosome lumen and degraded by resident hydrolases (proteases, lipases, nucleases and glucosidases). The resulting degradation products are released back into the cytosol through the activity of specific membrane permeases for re-use (Klionsky, 2005; Yang and Klionsky, 2010).

\section{Signal transduction pathways influencing ageing modulate autophagy}

\subsection{Insulin/IGF-1 signalling}

Reduced activity of the insulin/IGF-1 signalling pathway (IIS) extends lifespan in C. elegans, Drosophila and other multicellular organisms, establishing its evolutionarily conserved role during ageing (Fontana et al., 2010; Kenyon, 2005, 2010). In C. elegans, mutations in the insulin/IGF-1 receptor orthologue DAF-2 double animal lifespan. Likewise, mutations affecting the conserved phosphatidylinositol-3 kinase PI(3)K/AKT/PDK kinase cascade that acts downstream of DAF-2 also extend worm lifespan (Fig. 2). This increase in longevity depends on the activity of DAF-16, a Forkhead FoxO transcription factor (Kenyon et al., 1993). DAF-16 is also required for some aspects of a distinctive developmentally arrested diapause stage (the dauer larva) that is characterized by low metabolic activity and a long lifespan in response to adverse environmental conditions including starvation (Ogg et al., 1997).

Interestingly, increasing DAF-16/FoxO activity in the intestine of $C$. elegans, which serves as the animal's adipose tissue, can completely rescue the longevity of $d a f-16(-)$ germline-defective mutants and extend lifespan of daf-16(-); daf-2(-) mutants by $\sim 50 \%$, whereas DAF-16 activity in neurons accounts for only $5-20 \%$ of the lifespan extension in these animals. In addition, overexpression of DAF-16 in one tissue can upregulate DAF-16 activity in other cells. Together, these findings suggest that DAF-16 may control two types of downstream signals, one that does not require DAF16 activity in responding cells, and one that does. Furthermore, different tissues differ in their ability to send and respond to these signals (Libina et al., 2003). In the worm, the three distinct DAF-16 isoforms, $a, b$ and the recently identified $d / f$ functionally cooperate to modulate IIS-mediated longevity, stress response and dauer diapause. RNA interference targeting either isoform-specific or common cDNA sequences shows that both DAF-16 $a$ and DAF$16 \mathrm{~d} / \mathrm{f}$ are important regulators of longevity in C. elegans. These DAF-16 isoforms display different tissue-specific expression patterns, preferential regulation of their activity by the two upstream AKT kinases, with DAF-16 $d / f$ preferentially regulated by AKT-1, and distinct and overlapping regulation of target genes. Thus, C. elegans seems to use multiple isoforms of a single gene product to fine-tune IIS-mediated processes in the context of a whole organism (Kwon et al., 2010).

Similarly to DAF-16, the heat shock transcription factor, HSF-1 is required for lifespan extension by reduced insulin/IGF-1 signalling. Accumulating evidence suggests that HSF-1 and DAF-16 increase longevity at least in part by activating expression of specific genes, including genes encoding small heat-shock proteins (Hsu et al., 2003). Extension of lifespan by inhibiting IIS signalling also requires the orthologue of mammalian Nrf1/2/3/,SKN-1, which contributes to the stress resistance and longevity phenotypes of reduced IIS, and defines an important pro-longevity mechanism, acting through modulation of phase 2 detoxification gene expression (Tullet et al., 2008). SKN-1 functions in ASI chemosensory neurons which transmit longevity signals to increase lifespan by an endocrine mechanism (Bishop and Guarente, 2007b). Intriguingly, recent findings suggest that the misexpression of germline genes in somatic tissues contributes to the increased health and survival of daf-2 mutants. This germline like quality is most likely mediated by DAF-16 and SKN-1 (Curran et al., 2009).

Autophagy plays a prominent role in lifespan extension conferred by daf- 2 mutations in C. elegans. RNAi-mediated knockdown of the autophagy regulator bec-1 shortens the lifespan of long-lived daf-2 mutant animals (Kenyon, 2005; Melendez et al., 2003). Moreover, knockdown of the atg-7 and atg-12 C. elegans orthologues shortens the lifespan of both wild-type animals and daf-2 mutants (Hars et al., 2007). Consistent with these findings, loss-of-function mutations in bec-1, atg-18 and lgg-1 abrogate the long-lived phenotype of daf-2 mutants (Toth et al., 2008). Therefore, long-lived daf-2 mutants require both DAF-16 and autophagy for their longevity. In contrast to lifespan extension, daf-16 itself is not required for increased autophagy in daf-2 mutants. The fact that daf-16;daf-2 double mutants have the same high level of autophagy as do daf-2 single mutants, yet they are not long-lived suggests that autophagy may be insufficient to extend lifespan (Hansen et al., 2008) or autophagy may act downstream of DAF-16.

In Drosophila, reduced activity of IIS also extends lifespan, indicating that ageing is influenced by evolutionarily conserved pathways (Helfand and Rogina, 2003). Similarly to C. elegans, the Drosophila genome encodes a single insulin/IGF-1 receptor (dINR). Loss-of-function mutations in dINR or the insulin receptor substrate (IRS) chico increase life expectancy (Clancy et al., 2001; Tatar et al., 2001). Evidence also suggests that the fly dFoxO (the single Drosophila orthologue of DAF-16) is required for longevity. Mutations in FoxO reduce lifespan and stress resistance in Drosophila (Junger et al., 2003; Salih and Brunet, 2008). In addition, reduced survival of chico/foxo double mutants may occur in part because chico deficiency exacerbates the negative effects of foxo lossof-function alleles (Yamamoto and Tatar, 2011). As in worms, activating dFoxO in the cells of the fat body (the equivalent of mammalian white adipose and liver tissue) increases lifespan (Giannakou et al., 2004; Hwangbo et al., 2004). Accumulating findings suggest that cell-autonomous and non-cell-autonomous roles of insulin signalling combine to control ageing in Drosophila (Hwangbo et al., 2004). 


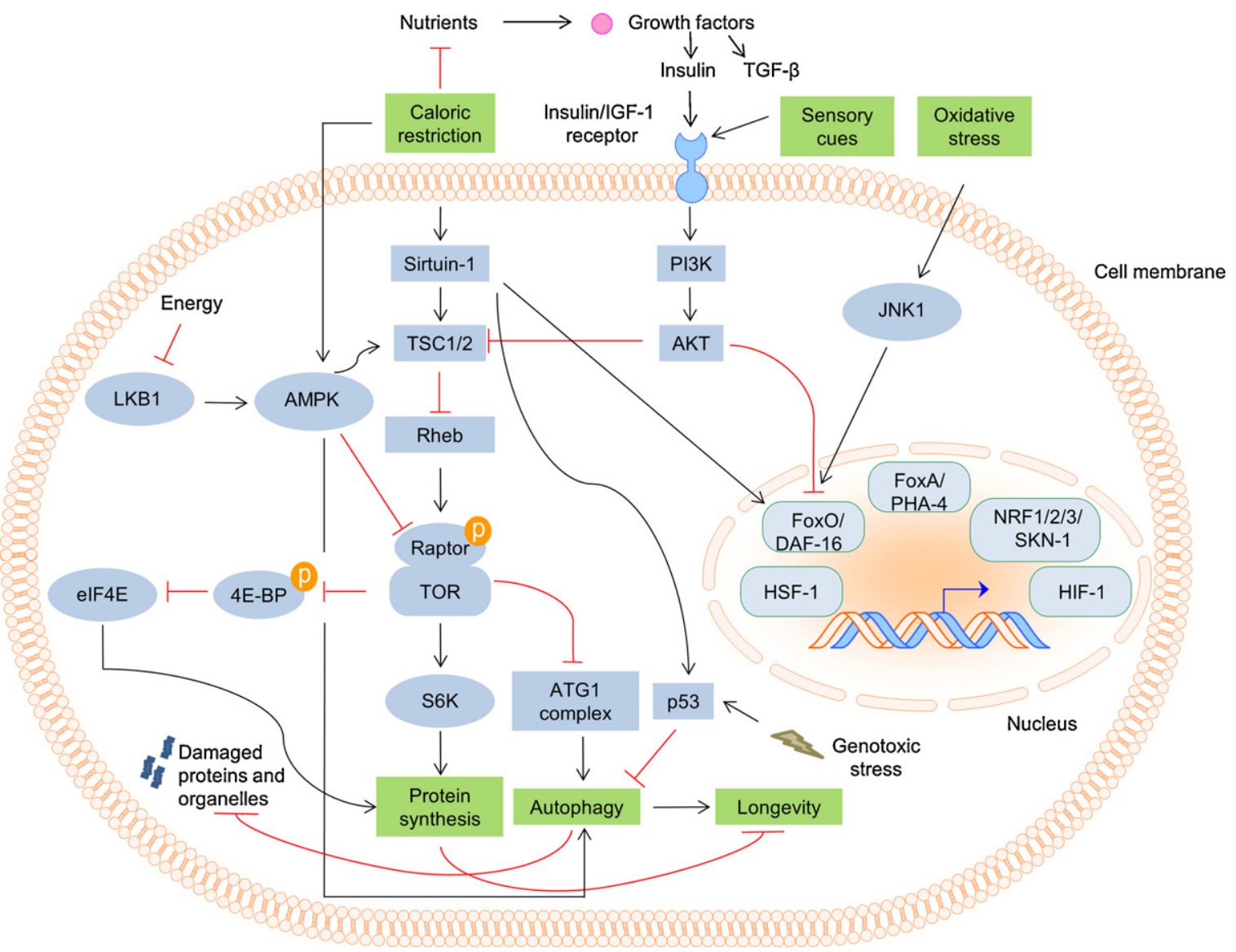

Fig. 2. Stress response pathways and signal transduction cascades that regulate longevity also interface with autophagy. Mutations inhibiting insulin/IGF-1 or TOR kinase signalling, depletion of p53, activation of sirtuin 1, hyperactivation of the stress-responsive JNK pathway and modest inhibition of mitochondrial respiration extend lifespan in a wide variety of species, including worms and flies (as well as yeast and mice, not shown). Similarly to these perturbations, limitation for nutrients, energy and growth factors, also augment longevity. Many of these pathways associate with autophagy regulation mechanisms. TOR plays a central role in the control of autophagy. Under nutrient abundance and elevated growth factor signalling, TOR promotes cell growth/metabolic activity and inhibits the Atg1 complex, causing suppression of autophagy. Under nutrient deprivation or stress, various signalling pathways inactivate TOR kinase activity and thus suppress cell growth, whilst inducing autophagy. Energy depletion activates the adenosine monophosphate-activated protein kinase (AMPK) which inhibits TOR, thus promoting autophagy. Black arrows indicate stimulatory inputs. Red bars indicate inhibitory interactions. For clarity, some of the signalling connections between longevity pathways and autophagy are not shown. See text for details. PI3K, phosphatidylinositol-3 kinase; TSC1/2, tuberosclerosis complexes 1 and 2; eIF4E, eukaryotic translation initiation factor 4E; eukaryotic initiation factor 4E-binding protein AKT, AKT8 virus proto-oncogene; JNK1, c-Jun N-terminal kinase; Rheb, Ras homologue enriched in brain; LKB1, serine/threonine protein kinase; S6K, S6 kinase; FOXO/DAF-16, a forkhead box O(FOXO) transcription factor; FOXA/PHA-4, a forkhead box A (FOXA) transcription factor; HSF-1, heat shock response transcription factor-1; NRF1/2/3/SKN-1, NF-E2-related factor/skin in excess transcription factor-1.

The mechanisms by which altered activity of FoxO transcription factors affects longevity have been a main focus of recent research efforts. In worms and flies, FoxO transcription factors promote longevity by regulating the expression of a series of target genes involved in metabolism, cell growth, cell proliferation, stress resistance and differentiation (Salih and Brunet, 2008). Recent evidence suggests that activation of FoxO and its target Thor/4E-BP in Drosophila muscles prevents muscle ageing and extends lifespan by regulating proteostasis in these cells. Interestingly, foxo overexpression in muscles increases the basal expression of several autophagy genes suggesting that $\mathrm{FoxO} / 4 \mathrm{E}-\mathrm{BP}$ signalling regulates protein homeostasis at least in part by enhancing the basal activity of the autophagy/lysosome system. In flies, FoxO/4E-BP signalling in muscles also decreases feeding behaviour and the release of insulin, which in turn delays age-related accumulation of protein aggregates in other tissues (Demontis and Perrimon, 2010).

\subsection{JNK and TGF- $\beta$ pathways}

The stress-responsive c-Jun N-terminal kinase (JNK), a component of mitogen-activated protein kinase (MAPK) cascade, and the transforming growth factor-beta (TGF- $\beta$ ) signalling pathways have also been implicated in ageing, in both worms and flies. In C. elegans, lifespan extension upon activation of the JNK pathway requires DAF-16 (Fig. 2). jnk-1 overexpression enhances the lifeextending effect of a daf-2 hypomorphic mutation, suggesting that JNK-1 regulates lifespan in parallel to the insulin-/IGF-1 pathway, with both pathways converging on DAF-16. JNK-1 regulates lifespan by directly interacting with DAF-16 and modulating its nuclear translocation (Oh et al., 2005). In Drosophila, JNK activation confers resistance to oxidative stress and extends the lifespan of adult flies by inducing the expression of protective genes (Wang et al., 2003). As in worms, dFoxO is required for JNK-mediated lifespan extension. JNK antagonizes IIS, causing nuclear localization of dFoxO and inducing the expression of dFoxO targets, including genes involved in stress response and growth control. Together, these findings suggest that FoxO is the convergence point of the opposing effects of IIS and JNK activity on longevity and stress response in Drosophila (Wang et al., 2005). Observations in Drosophila suggest that JNK protects from oxidative stress by inducing autophagy, at least in part through transcriptional activation of ATG (Wu et al., 2009). JNK-mediated stress resistance as well as induction of autophagy 
in flies deprived of food both require dFoxO (Luo et al., 2007; Wang et al., 2005; Juhasz et al., 2007b). Thus dFoxO may activate transcription of ATG in response to JNK signalling (Wu et al., 2009).

In addition to the insulin/IGF- 1 signalling pathway, the TGF- $\beta$ pathway regulates both dauer formation and adult lifespan in C. elegans. Similarly to the insulin/IGF- 1 pathway, TGF- $\beta$ signalling acts during adulthood to regulate longevity. Mutations that decrease the activity of the TGF- $\beta$ pathway significantly extend animal lifespan. Regulation of lifespan by TGF- $\beta$ requires DAF- 16 activity. The TGF$\beta$ and insulin/IGF-1 pathways share many transcriptional targets including DAF-16 target genes demonstrated to regulate longevity, suggesting that these pathways are more interconnected than previously thought (Shaw et al., 2007). Therefore, DAF-16/FoxO links insulin/IGF-1, TGF- $\beta$ and JNK signalling to regulate longevity. However, further studies are needed to establish whether autophagy mediates the effects of JNK gain-of-function or TGF- $\beta$ deficiency on longevity (reviewed in Vellai et al., 2009).

\subsection{TOR signalling}

The Target of Rapamycin (TOR) is a nutrient sensing, highly conserved signalling network that regulates cell growth and proliferation, development, metabolism and ageing in response to four major signalling cues: growth factors, nutrients, energy and stress (Rabinowitz and White, 2010; Wullschleger et al., 2006). S. cerevisiae harbours two homologous TOR genes whereas higher eukaryotes appear to have only one TOR gene. Eukaryote TORs are large proteins $(\sim 280 \mathrm{kDa})$ that belong to a group of kinases known as the phosphatidylinositol kinase-related kinase (PIKK) family. Members of PIKK family contain a carboxy-terminal serine/threonine protein kinase domain. Amino-terminal to the kinase domain is the FKB12-rapamycin binding domain. The central component of the TOR pathway is the TOR kinase, which participates in two structurally and functionally distinct multiprotein TOR complexes (TORC1 and TORC2); TORC1 is sensitive to rapamycin, whereas TORC2 is considered to be rapamycininsensitive, although in some cell lines, prolonged rapamycin treatment can also inhibit TORC2 activity (Sarbassov et al., 2005). Genetic and biochemical studies in yeast, C. elegans, Drosophila and mammalian cells have identified several upstream and downstream components of the TOR signalling pathway (Hay and Sonenberg, 2004).

In metazoa, TOR is essential for growth during early development, and also plays a critical role in the regulation of adult lifespan. Attenuation of TOR function in yeast, worms and flies results in a significant increase of lifespan (Kaeberlein et al., 2005; Kapahi et al., 2004; Martin and Hall, 2005; Vellai et al., 2003). TOR signalling controls various growth-related processes, including protein synthesis and autophagy, both of which are implicated in the modulation of lifespan. TOR positively regulates translation by activating the S6K kinase, a component of the small (40S) ribosomal subunit and inhibiting eIF4E binding proteins (4E-BPs; Fig. 2). Phosphorylated $4 \mathrm{E}-\mathrm{BP}$ releases eIF4E, which is then free to associate with eIF4G to promote translation initiation (Hay and Sonenberg, 2004; Tee and Blenis, 2005). TOR associates with S6K and 4E-BP via physical interaction between raptor (regulatory associated protein of mammalian TOR, mTOR) and a TOR signalling motif in S6K and 4E-BP (Nojima et al., 2003). Thus, TOR links nutrient availability to protein translation. Under reduced nutrient conditions, such as amino acid limitation, TOR activity is quenched, the TOR effectors S6K and $4 \mathrm{E}-\mathrm{BP}$ are dephosphorylated and consequently protein synthesis decreases. Reduction of mRNA translation by conditions that shift cells from states of nutrient utilization and growth to states of cell maintenance has been shown to increase lifespan of yeast, worm, flies and mice (Hansen et al., 2007; Harrison et al., 2009; Kapahi et al., 2004; Pan et al., 2007; Syntichaki et al., 2007).
In C. elegans, downregulation of the activity of CeTOR/let-363 or the TOR accessory protein raptor, DAF-15, extends adult lifespan (Vellai et al., 2003; Jia et al., 2004). Worms bearing mutations in S6K (RSKS-1) and components of the translational machinery such as ribosomal proteins and translation initiation factors, live longer (Hansen et al., 2007; Kaeberlein and Kennedy, 2008; Syntichaki et al., 2007). Recently, TORC2 has been implicated in regulation of worm longevity (Soukas et al., 2009). The PHA-4/FoxA transcription factor is required for adult lifespan extension in response to reduced CeTOR signalling. Although mutations in the RSKS-1/S6 kinase or in IFE-2/eIF4E reduce protein synthesis and increase lifespan, only RSKS- 1 mutations require PHA-4 for adult longevity (Sheaffer et al., 2008). Genetic studies in C. elegans indicate that TOR interacts with the insulin/IGF-1 signalling pathway to regulate ageing. As mentioned above, TOR, similarly to insulin/IGF-1 signalling acts during adulthood to influence ageing. However, the long-lived phenotype of let-363 RNAi worms is not suppressed by mutations in daf-16, indicating that TOR may be acting downstream or independently of DAF-16 (Jia et al., 2004; Vellai et al., 2003). Importantly, knockout of ife-2, one of five C. elegans eIF4E isoforms (ife-1 to ife-5), extends lifespan, and enhances resistance to oxidative stress, UV irradiation and heat shock independently of DAF-16/FoxO (Syntichaki et al., 2007).

In addition, TOR and specifically the TOR complex 1 (TORC1) is a key upstream regulator of autophagy that functions through inhibition of the Atg1 complex (Fig. 2). Despite overall evolutionary conservation, TOR-mediated modulation of autophagy displays some intriguing differences among eukaryotes. Under nutrientrich conditions, TOR-dependent phosphorylation of Atg 13 prevents complex assembly and thus inhibits autophagy in yeast. Starvation results in rapid de-phosphorylation of Atg13, assembly of the complex, increase in Atg1 kinase activity and induction of autophagy. Unlike in yeast and mammals however, Drosophila Atg13 is always associated with Atg1. Atg13 becomes hyper-phosphorylated during autophagy. TORC1 binds to the Atg1 complex when autophagy is induced in Drosophila. In well-fed Drosophila cells, overexpression of wild-type Atg1 leads to high levels of autophagy, indicating that Atg1 activity promotes not only the initial steps of autophagy induction, but can be sufficient to drive a full autophagic response in this system (reviewed by Neufeld, 2010).

In C. elegans, autophagy is required for the increased survival of TOR-deficient worms. Mutations in bec-1, unc-51, and atg-18 completely abrogate the long-lived phenotype of let-363/CeTOR(RNAi) adults, implying that the effect of TOR inhibition on lifespan is mediated by autophagy (Toth et al., 2008). Interestingly, RNAi knockdown of TOR in wild-type worms induces the expression of a predicted triglyceride lipase, LIPL-4/KO4A8.5. LIPL-4 upregulation is important for lifespan extension in germline deficient animals (Wang et al., 2008). These mutants display reduced TOR levels and increased autophagy. Moreover, autophagy is required for the increased longevity of germline-less animals. It seems plausible therefore that germline removal prolongs lifespan, at least in part, by reducing TOR signalling, which in turn upregulates both autophagy and lipolysis. PHA-4/FOXA mediates the transcriptional activation of autophagy genes in germline-less $g l p-1$ animals. Taken together, these findings suggest that autophagy and LIPL-4 interdependently modulate lifespan in germline-deficient $C$. elegans (Lapierre et al., 2011).

In Drosophila, inhibition of TOR signalling extends lifespan, as it does in yeast and in C. elegans (Kapahi et al., 2004; Bjedov et al., 2010). Flies mutant for TOR or overexpressing dominant negative forms of S6K, TSC1 or TSC2 (suppressors of the TOR pathway), are long-lived (Kapahi et al., 2004; Luong et al., 2006). When nutrients are abundant, Drosophila dTOR downregulates autophagy by binding to and phosphorylating Atg1/ULK1 (reviewed in Wullschleger et al., 2006). 
The TOR inhibitor rapamycin extends lifespan in yeast, worms, flies and mice (Bjedov et al., 2010; Hansen et al., 2008; Harrison et al., 2009; Kaeberlein et al., 2005; Kapahi et al., 2004; Toth et al., 2008). In yeast and in C. elegans, rapamycin only prolongs lifespan under conditions where autophagy can be induced (Alvers et al., 2009; Hansen et al., 2008). Rapamycin-induced autophagy is independent of the $\mathrm{NAD}^{+}$-dependent histone deacetylase sirtuin 1 . In Drosophila, rapamycin treatment during early adulthood extends lifespan, increases resistance to both starvation and paraquat, reduces female fecundity and significantly increases lipid levels. Analysis of the underlying mechanisms shows that the positive in vivo effects of rapamycin on lifespan are mediated by physiological processes downstream of TORC1. Rapamycin efficiently reduces S6K phosphorylation and activates 4E-BP. Thus, reduction of mRNA translation likely promotes longevity in this setting (Bjedov et al., 2010).

In addition to altering mRNA translation, rapamycin increases Drosophila lifespan by activating autophagy. Reduced autophagic activity during both Drosophila development and adulthood has been shown to shorten the lifespan of flies. This effect is sometimes associated with reduced protein aggregate clearance and degeneration in the adult nervous system (Juhasz et al., 2007a; Ravikumar et al., 2004; Simonsen et al., 2008; Toth et al., 2008). Atg5 depletion, which inhibits autophagy at the early membrane elongation/engulfment stage (Ren et al., 2009; Scott et al., 2004), completely abrogates the lifespan prolonging effect of rapamycin in adult Drosophila, indicating that upregulation of autophagy mediates longevity extension by rapamycin. Therefore, rapamycin increases adult fly lifespan through alterations of both mRNA translation and autophagy. Interestingly, rapamycin treatment further increases lifespan of weak insulin/IGF-1 signalling mutants and of flies with lifespan maximized under dietary restriction, suggesting that additional mechanisms may underlie the beneficial effects of rapamycin on longevity (Bjedov et al., 2010).

\subsection{AMP-activated protein kinase}

The AMP-activated protein kinase (AMPK) belongs to a conserved family of heterotrimeric eukaryotic kinases, consisting of a catalytic $\alpha$ subunit and regulatory $\beta$ and $\gamma$ subunits. AMPK functions as sensor of the cellular energy status, and becomes activated by high AMP/ATP ratio. In addition to acting at the level of the individual cell, the system also regulates food intake and energy expenditure at the whole body level (Hardie, 2004). How AMPK influences lifespan is not fully understood. In C. elegans, the AMPK $\alpha$ subunit (AMPK $\alpha 2$ ) AAK-2 is activated by AMP and functions to promote longevity. In addition, mutations in the aak-2 gene, coupled with mutations in daf-16 decrease worm lifespan further than single gene lesions do. These findings indicate that AAK-2 acts in parallel with DAF-16/FoxO to increase lifespan. AAK-2 may act as a sensor that couples energy availability information and insulinlike signals to influence lifespan (Apfeld et al., 2004). AMPK also mediates longevity in response to dietary restriction (DR; a reduction of food intake without malnutrition) in C. elegans. In this case, AMPK exerts its beneficial effects on lifespan in part by phosphorylating the DAF-16/FoxO and activating DAF-16/FoxO dependent transcription, suggesting that an energy-sensing AMPK-FoxO pathway is involved in the anti-ageing effects of DR (Greer et al., 2007). Whether autophagy is required for lifespan extension by AMPK remains unclear. Nevertheless, the finding that metabolic stress results in AMPK activation by AMP and the Peutz-Jeghers syndrome gene product LKB1, with subsequent induction of autophagy in human cancer cells (Fig. 2; Liang et al., 2007), raises the possibility that autophagy may mediate the effect of AMPK on lifespan in C. elegans.

\subsection{Sirtuins}

Sirtuins are nicotinamide adenine dinucleotide $\left(\mathrm{NAD}^{+}\right)$dependent protein deacetylases that function in gene expression regulation and maintenance of genome stability. The role of sirtuins in ageing was discovered in the yeast $S$. cerevisiae, where overexpression of Sir2 increases replicative lifespan (Kaeberlein et al., 1999). In higher eukaryotes, sirtuins have been implicated in ageing although their effect on lifespan remains controversial. Overexpression of sir-2.1 in C. elegans and dSir2 in Drosophila has been reported to extend lifespan (Tissenbaum and Guarente, 2001; Viswanathan et al., 2005; Rogina and Helfand, 2004). However, recent studies suggest that increased longevity observed in strains overexpressing sirtuin genes was due to unrelated mutations lurking in the genome and not to sirtuin overexpression per se. In C. elegans, outcrossing of a line with high level sir-2.1 overexpression abrogated the lifespan increase without diminishing sir-2.1 overexpression. Increased longevity of these transgenic animals has been attributed to an associated sensory defect. Similarly, although a Drosophila strain with ubiquitous overexpression of dSir2 mediated by a tubulin-GAL4 driver was long-lived relative to wild-type controls, as previously reported (Rogina and Helfand, 2004), it was not long-lived relative to appropriate transgenic controls. These findings imply that the effects of dSir2 overexpression on lifespan in flies could reflect heterosis in the vicinity of the transgene inserts or a mutagenic effect of transgene insertion (Burnett et al., 2011).

This apparent discrepancy may in part originate from variations in the genetic background among the different laboratory strains tested and/or fluctuations of the environmental conditions under which lifespan experiments are conducted. For example, it has been shown that diet can contribute to alterations in the longevity of flies and can significantly affect the metabolic profile, physiology and lifespan of worms (Reinke et al., 2010; Toivonen et al., 2007). Thus, the reported experimental inconsistencies reinforce the importance of rigorous control of genetic and environmental factors when studying the effects of single gene mutations on multifactorial quantitative traits like longevity. Collectively, these latest reports indicate that Sir2 overexpression is one of the genetic manipulations known to regulate ageing in invertebrates and suggest that Sir2 confers a modest lifespan extension in worms and flies. A recent study on mouse longevity shows that overexpression of SIRT6, one of the seven mammalian sirtuin homologues of the yeast Sir2, leads to a significant lifespan extension in male but not in female mice. Both sexes appeared to have improved glucose tolerance but only transgenic males displayed lower serum levels of insulin-like growth factor 1 (IGF1), higher levels of IGFbinding protein 1 and altered phosphorylation levels of major components of IGF1 signalling. These findings suggest that SIRT6 extends lifespan only in male mice potentially by reducing IGF1 signalling specifically in white adipose tissue (WAT; Kanfi et al., 2012). In addition, numerous studies report that sirtuins are crucial regulators of metabolic homeostasis and healthspan (reviewed in Houtkooper et al., 2012; Longo and Kennedy, 2006). Taken together, these studies suggest that although the role of sirtuins as sensu stricto longevity genes may be overestimated, sirtuins play important roles in regulating cellular stress responses, genomic stability and metabolism, major contributors to longevity.

The mechanisms that mediate the effects of sirtuins on ageing are not clear. In C. elegans, overexpression of sir-2.1 extends lifespan in a DAF-16/FoxO dependent manner. SIR-2.1 interacts with the worm 14-3-3-like proteins PAR-5 and FTT-2 to influence DAF16/FoxO activity and lifespan (Berdichevsky et al., 2006). Under stress, SIR-2.1 interacts with DAF-16 in a 14-3-3-dependent manner to facilitate stress resistance. Nevertheless, loss of sir-2.1 does not reduce the long lifespan of insulin/IGF-1 signalling-defective 
daf-2 mutants, indicating that sir-2.1 is not required for lifespan extension by reduced insulin/IGF-1 signalling. These findings suggest that SIR-2.1 and 14-3-3 act in parallel to the insulin/IGF-1 pathway to activate DAF-16 and extend lifespan.

Recent observations have highlighted a link between sirtuins and autophagy. Transgenic expression of sir-2.1 induces autophagy in C. elegans. Interestingly, deletion or depletion of Atg6/bec-1 not only suppresses the induction of autophagy by sir-2.1 but also abrogates longevity in these worms (Morselli et al., 2010a). In addition, activation of SIR-2.1 by transgenic overexpression of pnc-1, a gene encoding the pyrazinamidase/nicotinamidase PNC-1 that catabolizes nicotinamide, a sirtuin inhibitor, also extends lifespan in $C$. elegans. The effect of pnc-1 overexpression on lifespan is abolished by concomitant knockdown of sir-2.1 or by that of either bec-1 or atg-5 (Morselli et al., 2010b). Notably, SIRT1 (human orthologue of sir-2.1) has been shown to directly deacetylate Atg5, Atg7 and Atg8 in vitro (Lee et al., 2008), indicating that the transcriptional control of essential autophagy genes is under the control of SIRT1. Hence, SIRT1 appears to play an important role in autophagy regulation.

Sirtuin 1 has also been reported to mediate the beneficial effects of caloric restriction (CR) on lifespan (Fig. 2); however, the role of sirtuins in CR remains controversial. Deletion of $C$. elegans sir-2.1, combined with lesions in the eat-2 gene that genetically mimic CR, suppress the longevity of eat-2 mutant animals, indicating that sir-2.1 is required for CR-induced longevity (Wang and Tissenbaum, 2006). Consistent with these findings, a loss of function mutation in sir-2.1 prevents the induction of autophagy and the increase in longevity by caloric restriction, suggesting that CR induces autophagy in a sir-2.1-dependent manner. By contrast, rapamycin-induced autophagy is independent of sir-2.1, indicating that rapamycin and sir-2.1 stimulate autophagy through distinct mechanisms (Morselli et al., 2010b). Dietary deprivation (removal of food source Escherichia coli) during adulthood increases lifespan and enhances thermotolerance and resistance to oxidative stress in C. elegans. Unlike partial reduction of food availability, starvation-induced longevity (complete removal of bacterial food during adulthood), is independent of sir-2.1 and the insulin/IGF-1 signalling pathway (Lee et al., 2006; Kaeberlein et al., 2006).

In Drosophila, Sir2 has been reported to mediate the effect of caloric restriction-induced lifespan extension. In addition, caloric restriction does not further extend the lifespan of long-lived dSir2 overexpressing flies. Reduced dSir2 activity prevents lifespan extension by caloric restriction or mutations in rpd3 (another deacetylase gene), known to increase lifespan by acting within a pathway related to caloric restriction (Rogina and Helfand, 2004; Rogina et al., 2002). However, a recent study questions the conserved role of dSir2 in mediating lifespan extension in response to dietary restriction (Burnett et al., 2011).

\section{6. $p 53$}

$\mathrm{p} 53$, the "guardian of the genome" plays important roles in the maintenance of genomic integrity in response to cellular damage and stress (Lane, 1992). Inactivating mutations or deletions in p53 are among the most common events in human cancers (Levine, 1997). Recent findings also implicate p53 in the regulation of lifespan. Invertebrates such as worms and flies do not develop cancer upon deletion of p53 (Rutkowski et al., 2011). Nevertheless, p53 deficiency broadly affects development, metabolism, cell death, the response to genotoxic stress and the overall fitness of the organism, which may account for the impact of p53 on life expectancy (Fig. 2; Vousden and Lane, 2007).

C. elegans cep-1 mutants (the nematode orthologue of p53) are viable, although they show hypersensitivity to hypoxia-induced lethality and decreased lifespan in response to starvation-induced stress (Derry et al., 2001). Deletion, depletion or pharmacological inhibition of p53 induces autophagy in human, mouse and nematode cells. Enhanced autophagy improves the survival of p53deficient cancer cells under conditions of hypoxia and nutrient depletion, suggesting that autophagy induced by p53 inhibition has a cytoprotective role. Cytoplasmic, but not nuclear, p53 can repress the enhanced autophagy of p53-deficient cells, implying a complex role for p53 in regulation of autophagy (Fig. 2; Tasdemir et al., 2008). CEP-1 depletion extends lifespan in a DAF-16/FoxOdependent manner (Arum and Johnson, 2007; Ventura et al., 2009). Interestingly, knockdown of the essential autophagy gene bec-1 reduces the longevity of cep- 1 mutant animals, suggesting that the lifespan-extending effect of CEP-1 deficiency is mediated by autophagy (Tavernarakis et al., 2008). In addition, CEP-1 plays a critical role in both development and adult lifespan regulation, in response to mild mitochondrial stress. Partial loss of mitochondrial protein function extends lifespan in worms. This increase in longevity requires CEP-1. In sharp contrast, CEP-1 impairs development and shortens lifespan upon severe mitochondrial damage (Ventura et al., 2009). The mechanisms by which CEP-1 mediates longevity remain unclear.

Flies lacking Dmp53 (the Drosophila p53 homolog) are viable, and still undergo cell cycle arrest and apoptosis following DNA damage, but display abnormal germ cell death, similarly to $C$. elegans (Yamada et al., 2008). Dmp53 has also been implicated in ageing. Loss-of-function Dmp53 mutations shorten lifespan, probably due to negative effects during embryonic development. Surprisingly, expression of dominant-negative (DN) Dmp53 mutations in adult neurons extends lifespan by 58\% in females and 32\% in males, and increases genotoxic stress resistance in the fly (Bauer and Helfand, 2006). Furthermore, specific expression of DN Dmp53 in 14 brain neurons that produce insulin-like peptides prolongs lifespan to an extent comparable to pan-neuronal expression, suggesting that Dmp53 inhibition promotes longevity by inhibiting insulin signalling (Bauer et al., 2007). Caloric restriction does not further extend lifespan in DN Dmp53 expressing flies, suggesting that a decrease in p53 activity may mediate the longevity response to caloric restriction in flies. In Drosophila, the pro-longevity effects of DR have been reported to involve activation of dSir2 (Rogina and Helfand, 2004). Lifespan extension by both activation of dSir2 and DN Dmp53 expression is not additive. Moreover, dSir2 physically interacts with Dmp53 and can deacetylate Dmp53-derived peptides (Bauer et al., 2009). Collectively, these observations suggest that CR, dSir2 and Dmp53 may function in the same pathway to influence life expectancy.

\subsection{Calcineurin}

Calcineurin ( $\mathrm{CaN})$ is a serine/threonine phosphatase, activated by $\mathrm{Ca}^{2+} /$ calmodulin $\left(\mathrm{Ca}^{2+} / \mathrm{CaM}\right)$, playing a role in the coupling of $\mathrm{Ca}^{2+}$ to cellular responses (Klee and Haiech, 1980). It is a heterodimer consisting of a $60 \mathrm{kDa}$ catalytic subunit, calcineurin A ( $\mathrm{Cn} A$, encoded by tax- 6 in C. elegans) and a $19 \mathrm{kDa}$ calcium binding regulatory subunit, calcineurin $\mathrm{B}(\mathrm{CnB}$, encoded by $c n b-1$ in C. elegans). Calcineurin deficiency extends lifespan and enhances autophagy in the nematode. RNAi-mediated depletion of bec-1 and atg-7 genes suppresses lifespan extension in CaN mutants, indicating that autophagy is required for the longevity phenotype of calcineurin defective C. elegans strains (Dwivedi et al., 2009).

\section{Environmental/cellular stress signals induce autophagy-mediated lifespan extension}

Living organisms need to cope with multiple different environmental or chemical stressors (food deprivation, temperature shifts, UV irradiation, oxidation agents, etc.). Therefore, strategies that 
confer resistance to diverse stress stimuli are crucial for survival. In several cases, resistance to stress has been linked to longevity (Johnson et al., 2002; Kourtis and Tavernarakis, 2011). Stress resistance is often studied in two different contexts: survival after acute stress and adaptation to mild stress. The latter is often also manifested as hormesis (Martins et al., 2011). Hormesis is the adaptive response to small harmless doses of a potentially fatal environmental or intrinsic stress stimulus, which renders the organism resistant to increased doses of this particular stressor. The signalling pathways that mediate resistance or adaptation to stress are also involved in longevity and have been already introduced in previous chapters (Calabrese et al., 2011; Johnson et al., 2002; Parsons, 1995). In this part of the review, we highlight autophagy as an integral component of several stress response mechanisms, which represents a conserved stress coping strategy in eukaryotes.

\subsection{Autophagy and dauer formation in C. elegans}

C. elegans has developed a survival strategy for harsh environmental conditions that entails entering an alternate third developmental stage, the "dauer diapause". Dauer larvae do not eat or defecate, they store large amounts of fat in their intestine, which gives them an intensive intestinal pigmentation (Apfeld and Kenyon, 1998), and they survive for extended periods of time compared to reproductively active adult nematodes. When environmental conditions improve, dauer larvae exit diapause and continue their reproductive development. The decision of whether to enter diapause or proceed towards reproductive lifecycle is controlled by insulin/IGF-1 signalling (Kimura et al., 1997). daf-2 mutants, that lack the insulin-like receptor show a DAF-16/FoxOdependent, constitutive dauer phenotype at high temperature $\left(25^{\circ} \mathrm{C}\right)$ and live twice as long as wild-type animals (Kenyon et al., 1993; Lin et al., 1997). Autophagy is critical for both diapause entry and longevity. Essential autophagy genes are highly expressed in all tissues that are remodelled throughout dauer larval development (hypodermis, intestine, pharynx, nervous system, reproductive organs; Melendez and Levine, 2009). Accordingly, daf-2 mutants display increased levels of autophagy (Hansen et al., 2008). Normal dauer larvae are resistant to 1\% SDS (Cassada and Russell, 1975). Reduced autophagy results in the formation of abnormal dauer larvae with altered morphology, which lack resistance to SDS and are not long-lived (Hars et al., 2007; Melendez et al., 2003). Knockdown of the autophagy gene bec-1 shortens lifespan of both wild-type and daf-2 mutant worms. Orthologues of the yeast autophagy genes APG1, APG7, APG8, and AUT10 are also required for dauer formation and lifespan extension in C. elegans (Melendez et al., 2003). Collectively, these findings indicate that autophagy is required for the formation of stress resistant, long-lived dauer larvae, and is important for both normal lifespan and longevity induced by low endocrine signalling (Melendez et al., 2003).

\subsection{Autophagy and starvation}

Severe starvation or milder dietary restriction (DR) induces autophagy in several tissues of both invertebrate models. Indicatively, following DR, autophagy is stimulated in fat body, muscles and ovaries of Drosophila (Barth et al., 2011) and pharyngeal muscles of C. elegans (Kang et al., 2007). DR is the only environmental manipulation with a positive impact on the lifespan of all species tested so far. Does autophagy play a causative role in DR-mediated lifespan extension? In C. elegans, DR induces autophagy (Hansen et al., 2008). Knockdown of autophagy genes such as bec-1, vps-34 or atg-7, prevents lifespan extension under DR (Hansen et al., 2008; Jia and Levine, 2007; Morselli et al., 2010b). Accordingly, autophagy is required for lifespan extension of eat- 2 mutants that display pharyngeal pumping defects, and are thus chronically dietaryrestricted (Jia and Levine, 2007; Toth et al., 2008). Survival upon complete starvation also depends on autophagy. In Drosophila, starvation induces autophagy in the fat body (a nutrient storage organ), providing the nutrients required for survival (Scott et al., 2004). Under conditions of complete starvation or sugar-only diet, autophagy mutants, lacking the core autophagy regulator Atg7, display accelerated mortality rates compared to control flies (Juhasz et al., 2007a).

In C. elegans, lesions in pcm-1, a gene encoding a protein repair aminotransferase ( $\mathrm{L}$-isoaspartyl-O-methyltransferase), decrease dauer lifespan. Under complete starvation, $p c m-1$ mutant L2d larvae approaching the dauer molt, show reduced autophagy, and this likely underlies their inability to cope with starvation via dauer formation (Gomez et al., 2007). Importantly, unrestrained, excessive autophagy induced by over-activation of muscarinic acetylocholine receptor signalling in gpb-2 mutants reverses the protective effect of moderate autophagy and decreases survival upon starvation (Kang and Avery, 2008; Kang et al., 2007). In adult flies, conditional inactivation of autophagy genes does not have an effect on lifespan extension by DR (Ren et al., 2009). The PHA-4 and DAF-16 transcription factors mediate lifespan extension by DR in C. elegans (Honjoh et al., 2009; Panowski et al., 2007). Under limited food conditions autophagy is induced specifically by PHA-4 activation (Hansen et al., 2008). Whether DAF-16 activation also stimulates autophagy under intermitted fasting remains to be determined (Honjoh et al., 2009). Depletion of essential autophagy proteins, such as ATG1, may interfere with vital signalling pathways, such as the MTOR-S6 kinase pathway, which influence lifespan (Lee et al., 2007). A role for autophagy in starvation-induced longevity has also been reported in vertebrates (Hara et al., 2006; Komatsu et al., 2005).

\subsection{Autophagy and oxidative stress}

Intracellular oxidative stress is caused by increased levels of reactive free radical derivatives of oxygen and nitrogen species (ROS and RNS respectively; Milton and Sweeney, 2012). ROS and RNS are generated mainly as by-products of mitochondrial respiration, although they can also be produced by the ER (Liu et al., 2004), peroxisomes (Boveris et al., 1972), and lysosomes (Kurz et al., 2008). A mild inhibition of mitochondrial respiration has been shown to extend lifespan both in C. elegans and in Drosophila. Under normal conditions, an arsenal of cellular anti-oxidant systems (superoxide dismutase - SOD, catalase, glutathione - GSH, the thioredoxin system - TRX) protect against damage. Pathological conditions develop when the level of free radicals exceeds the reducing capacity of the cell. Oxidative damage is cumulative and its effects are exacerbated in post-mitotic cells, for example neural cells in Drosophila, or all somatic cells in C. elegans adults. The mitochondrial theory of ageing, proposed first by Harman (1956, 1972), posits that accumulating oxidative damage drives senescent decline and ageing. In C. elegans, long-lived daf-2 or isp-1 mutants display increased anti-oxidant enzyme expression (MnSOD and SOD-3 respectively; Feng et al., 2001; Honda and Honda, 1999), whilst short-lived gas-1 and mev-1 mutants show increased ROS levels as well as high mitochondrial and nuclear DNA mutation rates (Ishii et al., 1998; Kayser et al., 2004; Senoo-Matsuda et al., 2003). In Drosophila, the long-lived Methuselah (mth) mutants display increased resistance to oxidative stress (Lin et al., 1998), whilst overexpression of anti-oxidant enzymes extends lifespan (Sun et al., 2002; Sun and Tower, 1999). Paradoxically, knockdown of specific mitochondrial respiratory chain genes has a positive impact on nematode lifespan (Rea, 2005; Ventura et al., 2006). RNAi of five genes encoding respiratory chain components from complexes I, III, IV and V increases lifespan in flies (Copeland et al., 


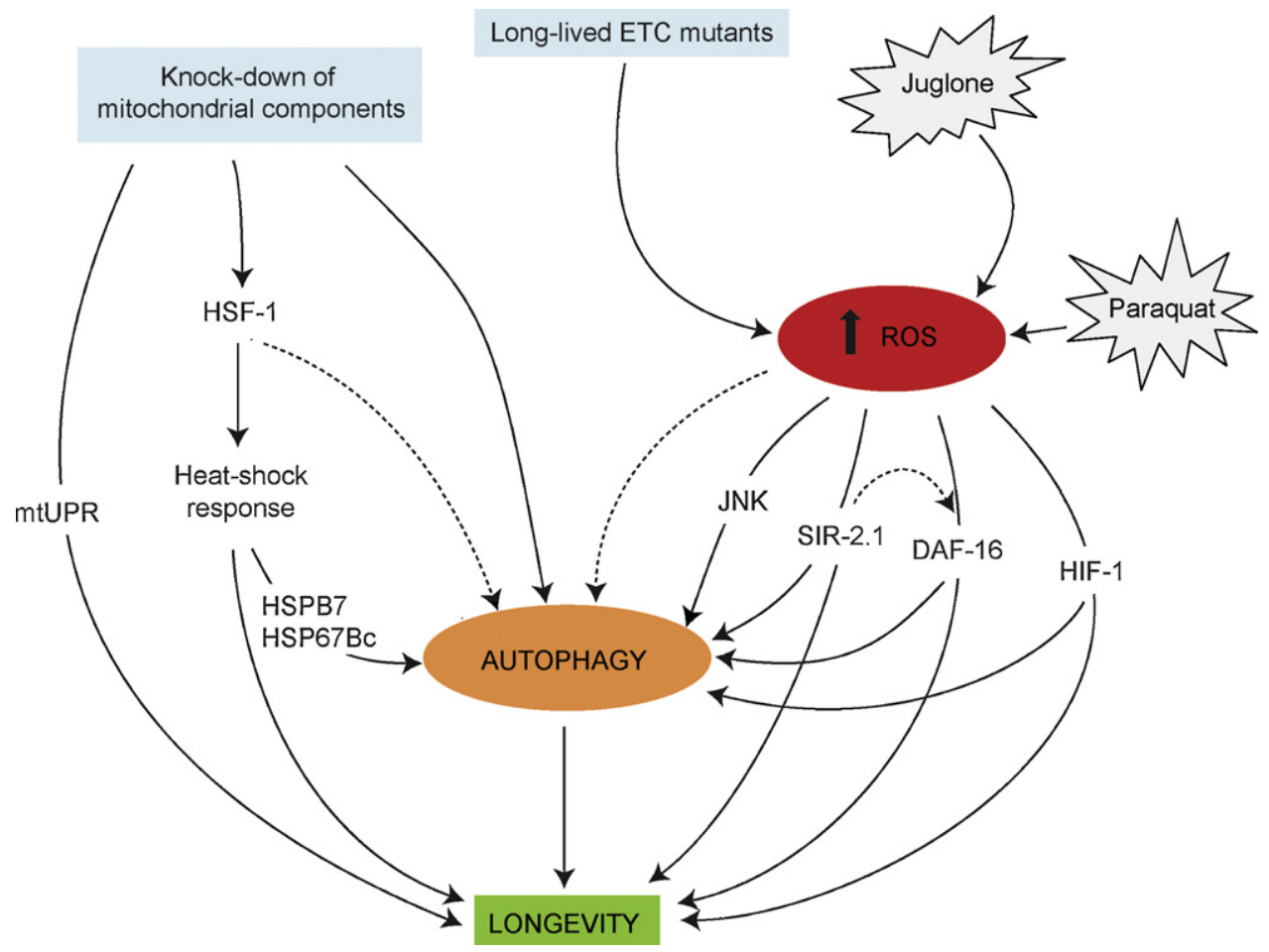

Fig. 3. Emerging interactions between autophagy, mitochondrial dysfunction and ROS production in the regulation of longevity. The schematic diagram illustrates pathways by which increased generation of ROS, caused either by mutations in ETC subunits or by treatment with oxidizing agents, likely promotes longevity. RNAi knockdown of mitochondrial subunits also induces longevity mediated by the heat-shock response and the mitochondrial unfolded protein response (mtUPR). Interestingly, most of these pathways converge to stimulate autophagy. Whether autophagy is the major mediator of longevity upon activation of these pathways remains to be investigated. Dashed lines indicate inferred but not yet unequivocally established interactions.

2009). Furthermore, targeted RNAi of two respiratory subunits in adult tissues or in neurons alone, is sufficient to extend lifespan in flies (Copeland et al., 2009).

Autophagy defects cause sensitivity to oxidative stress and decrease longevity. In C. elegans, autophagy is required for lifespan extension by low mitochondrial respiration upon knockdown of several mitochondrial electron transport chain (ETC) components (Toth et al., 2008). Mutations in autophagy genes (unc-51, bec-1 and atg-18) suppress lifespan extension by lesions in the mitochondrial ATP synthase gene atp-3 and the ubiquinone biosynthesis gene $c l k-1$ (Toth et al., 2008). Notably, the lifespan extension phenotype of isp-1 and nuo- 6 mitochondrial mutants does not depend on autophagy (Yang and Hekimi, 2010a). isp-1 and nuo-6 mutants are resistant to paraquat and express higher levels of SOD-1 and SOD-2, with no autophagy induction. Knockdown of bec-1 in adult worms does not suppress lifespan extension in isp-1 mutants (Hansen et al., 2008). Therefore, perturbation of mitochondrial function in C. elegans may trigger distinct longevity pathways, depending on the extent or the type of mitochondrial dysfunction (Fig. 3; Yang and Hekimi, 2010a).

In Drosophila, Atg7 mutants are hypersensitive to agents that induce oxidative stress (paraquat and hydrogen peroxide), and are short-lived compared to control flies under normal conditions, indicating the crucial role of autophagy in stress survival and longevity (Juhasz et al., 2007a). Similarly, flies with mutations in the Atg8a gene are short-lived, show accumulation of insoluble ubiquitinated proteins (IUP) and increased sensitivity to oxidative stress. By contrast, flies with elevated neuronal expression of Atg8a exhibit extended average lifespan and enhanced resistance to oxidative stress and the accumulation of ubiquitinated and oxidized proteins (Simonsen et al., 2008). The stress-induced protein kinase JNK mediates induction of autophagy in the Drosophila midgut, and resistance to oxidative stress by paraquat treatment (Fig. 3; Wang et al., 2003; Wu et al., 2009). Moreover, in the adult Drosophila brain, overexpression of autophagy genes augments resistance to oxidative stress and extends lifespan by $\sim 50 \%$ (Simonsen et al., 2008).

What signals induce autophagy upon mitochondrial dysfunction and oxidative stress? Recent findings, relevant to ROS levels in long-lived mitochondrial mutants are not directly compatible with the free radical theory of ageing. In contrast to the predictions of the theory, increased ROS levels do not always correlate with reduced longevity (Doonan et al., 2008). Paradoxically, ROS have been shown to even promote longevity in several cases, with anti-oxidants actually cancelling these beneficial effects (Fig. 3; Heidler et al., 2010; Schulz et al., 2007; Yang and Hekimi, 2010b). Consistently, long-lived clk-1, isp-1 and nuo-6 mitochondrial mutant worms have increased ROS levels (Lee et al., 2010; Yang and Hekimi, 2010b). Elevated ROS activate the hypoxiainducible factor-1 (HIF-1), which is required for lifespan extension in these mutants (Hwang and Lee, 2011; Lee et al., 2010). HIF-1 is a highly conserved transcription factor that mediates survival under hypoxic stress. Interestingly, genetic or pharmacological inhibition of autophagy causes hypoxia hypersensitivity in worms. Thus, autophagy appears to protect from hypoxic injury (Fig. 3; Samokhvalov et al., 2008). In flies, oxidative stress is a major regulator of synaptic function and growth by activation of JNK/AP1 and autophagy (Shen and Ganetzky, 2009). Such responses have been proposed to underpin some forms of synaptic ageing (Milton and Sweeney, 2012). Findings in yeast and mammalian cells suggest that mitochondrial ROS serve as signals to regulate induction of autophagy. For example, ROS activate redox-sensitive proteases that are involved in autophagy (Scherz-Shouval et al., 2007). Although, the exact link between ROS and the autophagic machinery remains largely unknown, it is tempting to speculate that elevated ROS may promote longevity by signalling autophagy induction. 


\subsection{Autophagy against heat shock and accumulation of protein aggregates}

Prokaryotes and eukaryotes exposed to elevated temperatures induce the expression of a battery of conserved genes encoding Heat-Shock Proteins (HSPs). HSPs function mainly as chaperons and proteases that target misfolded and aggregated proteins. This heat-shock response confers cyto-protection and stress resistance. Expression of heat-shock genes is orchestrated by the conserved transcription factor heat shock factor 1 (HSF1). Lack of HSF-1 expression causes shortening of lifespan in C. elegans (Garigan et al., 2002). Studies in mammals have also shown that the thermostability of HSF1 and ability to bind onto its respective DNA elements is altered with age (Heydari et al., 2000).

How does heat stress kill cells? Although the molecular mechanisms involved remain obscure, it is likely that loss of cell viability following heat shock is due in part to the formation of toxic protein aggregates, deriving from misfolded or heat-denatured proteins (Riezman, 2004). HSPs are modulators of stress resistance and ageing in flies (Tower, 2011). HSPs bind their substrates and either prevent their aggregation, catalyse their refolding or deaggregation, or target them for proteasomal or autophagic degradation (reviewed in Stetler et al., 2010). The ATP-independent small HSP family (HSPB) has been shown to mediate stress-induced macroautophagic processes (Vos et al., 2011). Amongst them HSPB7 is the most potent suppressor of polyQ aggregation, as shown both in mammalian cells and in Drosophila. HSPB7 binds polyQ protein aggregates but does not display refolding capacity. Although HSPB7 does not increase autophagy, it depends on an active autophagic machinery to perform its protective effects (Vos et al., 2010). Mutations in human HSPB8 have been associated with distal motor neuropathy (Irobi et al., 2004) and Charlott-Marie-Tooth (CMT) disease (motor and sensory neuropathy; Tang et al., 2005). HSP67Bc, the closest functional orthologue of HSPB8 in Drosophila, mediates the clearance of protein aggregates via downregulation of the mRNA translation initiation factor eIF2a. HSP67Bc-mediated phosphorylation of eIF2a leads to both suppression of protein synthesis and activation of autophagy (Carra et al., 2010), similarly to its mammalian counterpart (Carra et al., 2009). eIF2a inhibition is unrelated to ER stress, since it does not include the PERK kinase (see below; Carra et al., 2009). HSPB8 function depends on the interaction with BAG3, an autophagy stimulator (Carra et al., 2008). It has been hypothesized that BAG3 might recruit the autophagic machinery in close proximity to the cargo-loaded chaperone. The Drosophila orthologue of BAG3, Starvin could have a similar function (Carra et al., 2010). More autophagic machinery components could also be involved (Gamerdinger et al., 2009). However, apart from the initial evidence described above, the role of HSPs in regulating autophagy during ageing is yet to be discovered.

Oxidative and thermal stress, as well as aberrant gene products prone to aggregation, can lead to intracellular accumulation of misfolded or aggregated protein and proteotoxicity. Protein aggregates formed either in the ER or in mitochondria are sensed in these organelles and induce ER or mitochondria-specific unfolded protein responses (UPRs). UPRs involve the expression of ER and mitochondrial chaperones, and enzymes dedicated to repairing or eliminating misfolded proteins. Different sub-categories of HSPs participate in UPR pathways. In C. elegans, knockdown of $c c 0-1$, which encodes a cytochrome $c$ oxidase subunit, triggers the mitochondria UPR ( ${ }^{\mathrm{mt}} \mathrm{UPR}$ ), in a cell-non-autonomous manner, and also promotes longevity (Fig. 3; Durieux et al., 2011). In mammals, accumulation of misfolded proteins in the ER induces phosphorylation of the translation initiation factor eIF2a by the PERK kinase (pancreatic endoplasmic reticulum eIF2a kinase), a sensor of misfolded proteins in the ER, and also stimulates autophagy (Kouroku et al., 2007). In addition to ER stress, eIF2a phosphorylation is induced by other stress stimuli, such as amino-acid deprivation (via the GCN2 kinase), and the presence of dsRNA (via the PKR kinase). These eIF2a-phosphorylation pathways have been linked to autophagy induction in mammalian cell lines (Py et al., 2009; Talloczy et al., 2002).

Ageing weakens the capacity of the autophagic machinery to remove protein aggregates and maintain protein homeostasis (Fig. 3). In Drosophila, expression of several autophagy genes is reduced in the neurons of aged flies. Loss-of-function mutations in autophagy genes result in accumulation of insoluble ubiquitinated proteins in neurons and reduced lifespan (Simonsen et al., 2008). Moreover, natural brain ageing is accompanied by autophagic neuropathologies (accumulated dysfunctional autophagic/lysosomal vesicles), in Drosophila (Ling and Salvaterra, 2011). The involvement of autophagy in countering proteotoxicity is particularly relevant in age-related neurodegenerative diseases. Invertebrate models have provided valuable insights into the critical role of autophagy for clearance of protein aggregates that lead to disease. These findings, which hold promise for the development of effective therapeutic interventions, are discussed in Section 4.6, below.

\subsection{Mitophagy}

Apart from the general, non-selective autophagy, several types of selective autophagy have been characterized. Selective autophagy mediates the clearance of specific organelles that are either damaged or superfluous, under certain conditions. Depending on the specific cargo that is being degraded, distinct types of autophagy have been defined; pexophagy (degradation of peroxisomes), nucleophagy (degradation of the nucleus), mitophagy (degradation of mitochondria), ribophagy (for ribosomes) and xenophagy (for elimination of foreign particles, such as intracellular pathogens). Most of these forms of selected autophagy are largely unexplored in invertebrates (reviewed in Nezis, 2012). Here, we discuss mitophagy, one of the most studied process in invertebrates.

Mitochondria destined for elimination by mitophagy are marked by specific outer membrane markers (ATG32 in yeast, and NIX in mammalian cells) that mediate the conjugation to the isolation membrane (reviewed in Youle and Narendra, 2011). Damaged mitochondria, with disrupted membrane potential, are targeted to autophagosomes through the PINK-1/Parkin pathway (Jin and Youle, 2012). PINK1 is a mitochondrial-targeted kinase (Clark et al., 2006; Gandhi et al., 2006; Valente et al., 2004), whilst Parkin is an E3 ubiquitin ligase which seems to be specifically recruited to mitochondria upon PINK1 activation (Clark et al., 2006; Pallanck and Greenamyre, 2006). Several PINK1 (PTEN-induced putative kinase 1) and Parkin mutations have been detected in sporadic cases of Parkinson's disease patients (Ibanez et al., 2006). In flies, PINK1 mutants have reduced ATP levels, they are sensitive to multiple stresses, and have an overall shorter lifespan than wild-type controls (Clark et al., 2006), indicating the significance of mitochondrial quality control in ageing.

Studies in both Drosophila and mammalian cell lines suggest that PINK1 is stabilized on the outer membrane of uncoupled mitochondria and recruits Parkin. Subsequently, mitofusin (Mfn; a profusion factor on the outer mitochondrial membrane) is ubiquitinated in a Parkin-dependent manner for degradation by the proteasome (Narendra et al., 2010; Ziviani et al., 2010). Decreasing the levels of Mfn via the PIKN1/Parkin pathway promotes mitochondrial fission, or alternatively inhibits mitochondrial fusion (Poole et al., 2008, 2010; Yang et al., 2008). It is suggested that fragmentation of mitochondria that follows degradation of Mfn, segregates damaged mitochondria from the mitochondrial network and facilitates their subsequent engulfment by autophagosomes (Tanaka, 2010; Twig et al., 2008). These studies provide new insight into the 
complex interplay between mitochondrial dynamics and mitochondria quality control. The implications to lifespan determination remain to be elucidated.

\subsection{Autophagy against disease}

Expanded polyglutamine repeat proteins (poly-Q) aggregate intracellularly in Huntington' s disease and other polyQ disorders. Frequently, expanded polyglutamine tracts longer than 35-40 residues lead to neurodegeneration. In a $C$. elegans model for polyQ-induced cellular pathology, reduction of autophagy results in accumulation of polyQ aggregates and exacerbates muscle dysfunction, in animals expressing 40-residue long polyglutamine tracts in muscle cells (Jia et al., 2007). In addition, reduction of autophagy in the ASH sensory neurons of animals expressing a human huntingtin fragment (htt-polyQ150) enhances neurodegeneration (Jia et al., 2007).

Puromycin resistant aminopeptidase (PSA) cleaves polyQ sequences, both in mice and Drosophila. PSA overexpression reduces neuronal degeneration and extends lifespan in flies that express expanded huntingtin. Protection is likely mediated by autophagy (Menzies et al., 2010). In a Drosophila model for spinobulbar muscular atrophy (SBMA) that expresses an expanded polyQ androgen receptor (AR) variant, ubiquitin-positive protein aggregates accumulate in neurons. Autophagy is activated as a mechanism compensatory to the ubiquitin-proteasome system (UPS), in histone deacetylase 6 (HDAC6)-dependent manner, to protect against degeneration (Pandey et al., 2007). Drosophila dynein mutants with impaired autophagosome-lysosome fusion display premature expanded Huntingtin aggregation (Ravikumar et al., 2005; Rubinsztein et al., 2005). In a C. elegans model for Alzheimer's disease, expressing the human $\beta$-amyloid peptide $(A \beta)$, inhibition of autophagy exacerbates $A \beta$ toxicity, especially in genetic backgrounds deficient for insulin/IGF-1 signalling (Florez-McClure et al., 2007). Moreover, expression of human amyloid peptide $42\left(A \beta_{1-42}\right)$, but not $A \beta_{1-40}$, induces early onset and progressive neuropathology characterized by defective autophagic/lysosomal degradation (accumulation of dysfunctional autophagic vesicles) in the Drosophila brain (Ling and Salvaterra, 2011).

Protein aggregation is also a prominent feature of Parkinson's disease, where neuronal inclusions are formed called Lewy Bodies, which contain mostly aggregated $\alpha$-synuclein. $\alpha$-synuclein inhibits autophagy by blocking Rab1a function. Overexpression of Rab1a restores autophagy defect caused by $\alpha$-synuclein in the fly brain (Winslow et al., 2010). Collectively, these findings suggest that autophagy is a major cytoprotective mechanism against proteotoxic stress that lies at the cross-roads between ageing and age-related neurodegenerative diseases (reviewed in Markaki and Tavernarakis, 2010; McPhee and Baehrecke, 2009).

\section{Pharmacological induction of lifespan extension through autophagy stimulation}

Apart from rapamycin (discussed above), many other pharmacological interventions influence longevity or stress resistance in diverse species, through activation of the autophagic machinery. Resveratrol is a plant polyphenol, member of a polyphenol class known as flavonols. It is found in grape berry skin, red wine, knotweed, peanuts and other plants, and displays anti-oxidative and free-radical scavenging properties. It has been studied extensively for its anti-inflammatory (Zhang et al., 2010), anti-cancer (Jang et al., 1997) and antiviral roles (Yiu et al., 2010). Resveratrol has been reported to exert positive effects on lifespan in both D. melanogaster and C. elegans (Bass et al., 2007; Wood et al., 2004). The capacity of resveratrol to stimulate autophagy in C. elegans is mediated by sirtuin-1 (SIR-2.1; Morselli et al., 2010a,b).
Overexpression of SIR-2.1 induces autophagy in nematodes (Morselli et al., 2010a,b). Lifespan extension induced by resveratrol requires autophagy; inhibition of the autophagic machinery (by bec-1 knockdown) abolishes longevity (Morselli et al., 2010b). Lifespan extension by sirtuin 1 overexpression is also reversed in bec-1 autophagy deficient mutants (Morselli et al., 2010b). These findings indicate that resveratrol extends lifespan by inducing autophagy in a SIR-2.1-dependent manner. Moreover, resveratrol seems to protect the nematodes from oxidative stress caused by irradiation, by lowering ROS levels and preventing mitochondrial damage (Ye et al., 2010).

Spermidine is a naturally occurring ubiquitous polyamine. It is produced by prutrescine and is the precursor of spermine. Exogenous supply of spermidine induces lifespan extension in several organisms, including yeast, worms and flies. Spermidine also induces autophagy in all of these organisms and in human tumour cells (Eisenberg et al., 2009; Madeo et al., 2010; Morselli et al., 2011). Adding optimal doses of spermidine supplement in fly food, markedly increases their mean lifespan by about 30\%. Detected intracellular levels of spermidine and putrescine (interconvertible) are increased compared to control flies, indicating that exogenously supplied spermidine is indeed internalized and metabolized by the flies (Eisenberg et al., 2009). Similarly, spermidine supplied in food increases lifespan in C. elegans by $15 \%$. This pro-longevity effect is observed in both wild-type and sir-2.1 mutant animals (although sir-2.1 lesions attenuate autophagy induction by spermidine), suggesting that, in contrast to resveratrol, lifespan extension by spermidine is independent of sir-2.1 (Morselli et al., 2011). Interfering with the autophagic machinery, by knocking down Atg7 in flies or bec-1 in worms, abolishes the beneficial effects of spermidine on lifespan for both species (Eisenberg et al., 2009). In yeast, spermidine functions as an inhibitor of histone acetylases. Modulation of the acetylation state of histones affects the transcription of several genes, some of which are involved in the autophagic degradation machinery (Eisenberg et al., 2009). Although resveratrol induces autophagy through SIR-2.1, which is dispensable for autophagy induction by spermidine, clearly these two compounds mediate longevity through convergent pathways. Proteomic analysis of human colon carcinoma HCT116 cells revealed that the two pathways are both AMPK and mTOR independent, whilst they induce convergent acetylproteome modifications that regulate the autophagic network (Morselli et al., 2011).

Efforts to identify new autophagy promoting drugs revealed three new small molecules (SMERs) that can effectively activate autophagy both in mammalian cell lines and in Drosophila, without inhibiting the mTOR pathway (Sarkar et al., 2007). Autophagy induction by these agents protects against polyQ toxicity in neurons of an HD Drosophila model. Finally, amyloid-binding compounds, which also bind protein fibrils and protein aggregates, have been shown to influence lifespan in C. elegans. Interfering with autophagic or lysosomal function by RNAi knockdown of atg- 9 or $v s p-34$, partially reverses the beneficial effects of these compounds, indicating that their effects are mediated, at least in part, by the autophagy degradation pathway (Alavez et al., 2011).

\section{Concluding remarks}

The process of ageing is driven by the gradual, lifelong accumulation of a wide assortment of molecular and cellular damage that eventually results in frailty and disease (Kirkwood, 2005, 2008). Autophagy is a cellular housekeeper involved in the elimination of injured or dysfunctional organelles, protein aggregates and intracellular pathogens. These functions are vital for protection against damage associated with ageing and age-associated diseases (Levine and Kroemer, 2008). Survival under stress depends substantially 
on the ability of the autophagic machinery to remove damaged cellular components and reorganize the cell's metabolic strategy (Rabinowitz and White, 2010). Autophagic activity declines with age in vivo and in vitro, whilst deregulation of autophagy often correlates with accelerated ageing (Rubinsztein et al., 2011). Therefore, autophagy may exert a cytoprotective role by increasing the threshold of damage a cell can endure, prior to undergoing cell death (Martins et al., 2011). Somewhat paradoxically, RNAi suppression of several autophagy genes after development results in extended lifespan in worms (Hashimoto et al., 2009). Moreover, conditional inhibition of autophagy in adult flies did not affect lifespan (Bjedov et al., 2010). These findings argue for a protective role of autophagy against ageing specifically during development. Future work should elucidate this intriguing interplay between autophagy regulation during developmental and longevity.

In addition to its cytoprotective role, autophagy may contribute to cellular pathology when aberrantly or excessively activated (Kang and Avery, 2010). Inhibition of autophagy suppresses necrotic neurodegeneration, whilst over-induction of autophagy exacerbates necrotic cell death in C. elegans (Samara et al., 2008; Toth et al., 2007). Interestingly, the mouse anti-apoptotic protein Bcl-2 binds Beclin-1 and inhibits autophagy, whereas the proapoptotic BH3-only proteins release Beclin- 1 from Bcl-2, activating autophagy (Pattingre et al., 2005). Beclin-1 itself contains a BH3only protein domain; however, its interaction with Bcl-2 does not deprive Bcl-2 of its anti-apoptotic function (Boya and Kroemer, 2009; Ciechomska et al., 2009). Collectively these findings highlight the intricate relationship of autophagy with cellular homeostasis and organismal survival.

Although a tight link between autophagy and ageing has been established through numerous studies, a one-way cause-and-effect relationship still remains ambiguous. Ageing is a complex multifactorial process of progressive decay and physiological function decline. Autophagy is engaged in several situations and signalling events relevant to ageing and senescence. Further studies should reveal the complete repertoire of interactions between autophagy and ageing. An additional important challenge for the future that is particularly relevant to human age-related pathologies is the systemic, spatiotemporal regulation of autophagy, within different tissues and organs, in the context of the whole ageing individual.

\section{Acknowledgements}

Work in the authors' laboratory is funded by grants from the European Research Council (ERC), the European Commission Framework Programmes, and the Greek Ministry of Education.

\section{References}

Alavez, S., Vantipalli, M.C., Zucker, D.J., Klang, I.M., Lithgow, G.J., 2011. Amyloidbinding compounds maintain protein homeostasis during ageing and extend lifespan. Nature 472, 226-229.

Alvers, A.L., Wood, M.S., Hu, D., Kaywell, A.C., Dunn Jr., W.A., Aris, J.P., 2009 Autophagy is required for extension of yeast chronological life span by rapamycin. Autophagy 5, 847-849.

Apfeld, J., Kenyon, C., 1998. Cell nonautonomy of C. elegans daf-2 function in the regulation of diapause and life span. Cell 95, 199-210.

Apfeld, J., O'Connor, G., McDonagh, T., DiStefano, P.S., Curtis, R., 2004. The AMPactivated protein kinase AAK-2 links energy levels and insulin-like signals to lifespan in C. elegans. Genes and Development 18, 3004-3009.

Arum, O., Johnson, T.E., 2007. Reduced expression of the Caenorhabditis elegans p53 ortholog cep- 1 results in increased longevity. Journals of Gerontology. Series A Biological Sciences and Medical Sciences 62, 951-959.

Barth, J.M., Szabad, J., Hafen, E., Kohler, K., 2011. Autophagy in Drosophila ovaries is induced by starvation and is required for oogenesis. Cell Death and Differentiation 18, 915-924.

Bass, T.M., Weinkove, D., Houthoofd, K., Gems, D., Partridge, L., 2007. Effects of resveratrol on lifespan in Drosophila melanogaster and Caenorhabditis elegans. Mechanisms of Ageing and Development 128, 546-552.

Bauer, J.H., Helfand, S.L., 2006. New tricks of an old molecule: lifespan regulation by p53. Aging Cell 5, 437-440.
Bauer, J.H., Chang, C., Morris, S.N., Hozier, S., Andersen, S., Waitzman, J.S., Helfand, S.L., 2007. Expression of dominant-negative Dmp53 in the adult fly brain inhibits insulin signaling. Proceedings of the National Academy of Sciences of the United States of America 104, 13355-13360.

Bauer, J.H., Morris, S.N., Chang, C., Flatt, T., Wood, J.G., Helfand, S.L., 2009. dSir2 and Dmp53 interact to mediate aspects of CR-dependent lifespan extension in $D$. melanogaster. Aging (Albany, NY) 1, 38-48.

Berdichevsky, A., Viswanathan, M., Horvitz, H.R., Guarente, L., 2006. C. elegans SIR2.1 interacts with 14-3-3 proteins to activate DAF-16 and extend life span. Cell $125,1165-1177$.

Bishop, N.A., Guarente, L., 2007a. Genetic links between diet and lifespan: shared mechanisms from yeast to humans. Nature Reviews Genetics 8, 835-844.

Bishop, N.A., Guarente, L., 2007b. Two neurons mediate diet-restriction-induced longevity in C. elegans. Nature 447, 545-549.

Bjedov, I., Toivonen, J.M., Kerr, F., Slack, C., Jacobson, J., Foley, A., Partridge, L., 2010 Mechanisms of life span extension by rapamycin in the fruit fly Drosophila melanogaster. Cell Metabolism 11, 35-46.

Boveris, A., Oshino, N., Chance, B., 1972. The cellular production of hydrogen peroxide. Biochemical Journal 128, 617-630.

Boya, P., Kroemer, G., 2009. Beclin 1: a BH3-only protein that fails to induce apoptosis. Oncogene $28,2125-2127$

Burnett, C., Valentini, S., Cabreiro, F., Goss, M., Somogyvari, M., Piper, M.D., Hoddinott, M., Sutphin, G.L., Leko, V., McElwee, J.J., Vazquez-Manrique, R.P., Orfila, A.M., Ackerman, D., Au, C., Vinti, G., Riesen, M., Howard, K., Neri, C., Bedalov, A. Kaeberlein, M., Soti, C., Partridge, L., Gems, D., 2011. Absence of effects of Sir2 overexpression on lifespan in C. elegans and Drosophila. Nature 477, 482-485.

Calabrese, V., Cornelius, C., Cuzzocrea, S., Iavicoli, I., Rizzarelli, E., Calabrese, E.J., 2011. Hormesis, cellular stress response and vitagenes as critical determinants in aging and longevity. Molecular Aspects of Medicine 32, 279-304.

Carra, S., Seguin, S.J., Lambert, H., Landry, J., 2008. HspB8 chaperone activity toward poly(Q)-containing proteins depends on its association with Bag3, a stimulator of macroautophagy. Journal of Biological Chemistry 283, 1437-1444.

Carra, S., Brunsting, J.F., Lambert, H., Landry, J., Kampinga, H.H., 2009. HspB8 participates in protein quality control by a non-chaperone-like mechanism that requires eIF2\{alpha\} phosphorylation. Journal of Biological Chemistry 284 5523-5532.

Carra, S., Boncoraglio, A., Kanon, B., Brunsting, J.F., Minoia, M., Rana, A., Vos, M.J., Seidel, K., Sibon, O.C., Kampinga, H.H., 2010. Identification of the Drosophila ortholog of HSPB8: implication of HSPB8 loss of function in protein folding diseases. Journal of Biological Chemistry 285, 37811-37822.

Cassada, R.C., Russell, R.L., 1975. The dauerlarva, a post-embryonic developmental variant of the nematode Caenorhabditis elegans. Developmental Biology 46, 326-342.

Chen, Y., Klionsky, D.J., 2011. The regulation of autophagy - unanswered questions. Journal of Cell Science 124, 161-170.

Ciechomska, I.A., Goemans, G.C., Skepper, J.N., Tolkovsky, A.M., 2009. Bcl-2 complexed with Beclin-1 maintains full anti-apoptotic function. Oncogene 28, 2128-2141.

Clancy, D.J., Gems, D., Harshman, L.G., Oldham, S., Stocker, H., Hafen, E., Leevers, S.J., Partridge, L., 2001. Extension of life-span by loss of CHICO, a Drosophila insulin receptor substrate protein. Science 292, 104-106.

Clark, I.E., Dodson, M.W., Jiang, C., Cao, J.H., Huh, J.R., Seol, J.H., Yoo, S.J., Hay, B.A., Guo, M., 2006. Drosophila pink1 is required for mitochondrial function and interacts genetically with parkin. Nature 441, 1162-1166.

Copeland, J.M., Cho, J., Lo Jr., T., Hur, J.H., Bahadorani, S., Arabyan, T., Rabie, J., Soh, J. Walker, D.W., 2009. Extension of Drosophila life span by RNAi of the mitochondrial respiratory chain. Current Biology 19, 1591-1598.

Curran, S.P., Wu, X., Riedel, C.G., Ruvkun, G., 2009. A soma-to-germline transformation in long-lived Caenorhabditis elegans mutants. Nature 459, 1079-1084.

Demontis, F., Perrimon, N., 2010. FOXO/4E-BP signaling in Drosophila muscles regulates organism-wide proteostasis during aging. Cell 143, 813-825.

Derry, W.B., Putzke, A.P., Rothman, J.H., 2001. Caenorhabditis elegans p53: role in apoptosis, meiosis, and stress resistance. Science 294, 591-595.

Doonan, R., McElwee, J.J., Matthijssens, F., Walker, G.A., Houthoofd, K., Back, P., Matscheski, A., Vanfleteren, J.R., Gems, D., 2008. Against the oxidative damage theory of aging: superoxide dismutases protect against oxidative stress but have little or no effect on life span in Caenorhabditis elegans. Genes and Development $22,3236-3241$

Durieux, J., Wolff, S., Dillin, A., 2011. The cell-non-autonomous nature of electron transport chain-mediated longevity. Cell 144, 79-91.

Dwivedi, M., Song, H.O., Ahnn, J., 2009. Autophagy genes mediate the effect of calcineurin on life span in C. elegans. Autophagy 5, 604-607.

Eisenberg, T., Knauer, H., Schauer, A., Buttner, S., Ruckenstuhl, C., Carmona-Gutierrez, D., Ring, J., Schroeder, S., Magnes, C., Antonacci, L., Fussi, H., Deszcz, L., Hartl, R. Schraml, E., Criollo, A., Megalou, E., Weiskopf, D., Laun, P., Heeren, G., Breitenbach, M., Grubeck-Loebenstein, B., Herker, E., Fahrenkrog, B., Frohlich, K.U., Sinner, F., Tavernarakis, N., Minois, N., Kroemer, G., Madeo, F., 2009. Induction of autophagy by spermidine promotes longevity. Nature Cell Biology 11 1305-1314.

Feng, J., Bussiere, F., Hekimi, S., 2001. Mitochondrial electron transport is a key determinant of life span in Caenorhabditis elegans. Developmental Cell 1, 633-644.

Florez-McClure, M.L., Hohsfield, L.A., Fonte, G., Bealor, M.T., Link, C.D., 2007. Decreased insulin-receptor signaling promotes the autophagic degradation of beta-amyloid peptide in C. elegans. Autophagy 3, 569-580.

Fontana, L., Partridge, L., Longo, V.D., 2010. Extending healthy life span-from yeast to humans. Science $328,321-326$. 
Gamerdinger, M., Hajieva, P., Kaya, A.M., Wolfrum, U., Hartl, F.U., Behl, C., 2009. Protein quality control during aging involves recruitment of the macroautophagy pathway by BAG3. EMBO Journal 28, 889-901.

Gandhi, S., Muqit, M.M., Stanyer, L., Healy, D.G., Abou-Sleiman, P.M., Hargreaves, I., Heales, S., Ganguly, M., Parsons, L., Lees, A.J., Latchman, D.S., Holton, J.L., Wood, N.W., Revesz, T., 2006. PINK1 protein in normal human brain and Parkinson's disease. Brain: A Journal of Neurology 129, 1720-1731.

Garigan, D., Hsu, A.L., Fraser, A.G., Kamath, R.S., Ahringer, J., Kenyon, C., 2002. Genetic analysis of tissue aging in Caenorhabditis elegans: a role for heat-shock factor and bacterial proliferation. Genetics 161, 1101-1112.

Giannakou, M.E., Goss, M., Junger, M.A., Hafen, E., Leevers, S.J., Partridge, L., 2004. Long-lived Drosophila with overexpressed dFOXO in adult fat body. Science 305, 361.

Giannakou, M.E., Partridge, L., 2007. Role of insulin-like signalling in Drosophila lifespan. Trends in Biochemical Sciences 32, 180-188.

Gomez, T.A., Banfield, K.L., Trogler, D.M., Clarke, S.G., 2007. The L-isoaspartyl-Omethyltransferase in Caenorhabditis elegans larval longevity and autophagy. Developmental Biology 303, 493-500.

Greer, E.L., Dowlatshahi, D., Banko, M.R., Villen, J., Hoang, K., Blanchard, D., Gygi, S.P., Brunet, A., 2007. An AMPK-FOXO pathway mediates longevity induced by a novel method of dietary restriction in C. elegans. Current Biology 17, 1646-1656.

Guarente, L., Kenyon, C., 2000. Genetic pathways that regulate ageing in model organisms. Nature 408, 255-262.

Hansen, M., Taubert, S., Crawford, D., Libina, N., Lee, S.J., Kenyon, C., 2007. Lifespan extension by conditions that inhibit translation in Caenorhabditis elegans. Aging Cell 6, 95-110.

Hansen, M., Chandra, A., Mitic, L.L., Onken, B., Driscoll, M., Kenyon, C., 2008. A role for autophagy in the extension of lifespan by dietary restriction in $C$. elegans. PLoS Genetics 4, e24.

Hara, T., Nakamura, K., Matsui, M., Yamamoto, A., Nakahara, Y., Suzuki-Migishima, R., Yokoyama, M., Mishima, K., Saito, I., Okano, H., Mizushima, N., 2006. Suppression of basal autophagy in neural cells causes neurodegenerative disease in mice. Nature 441, 885-889.

Hardie, D.G., 2004. The AMP-activated protein kinase pathway-new players upstream and downstream. Journal of Cell Science 117, 5479-5487.

Harman, D., 1956. Aging: a theory based on free radical and radiation chemistry. Journal of Gerontology 11, 298-300.

Harman, D., 1972. The biologic clock: the mitochondria. Journal of the American Geriatrics Society 20, 145-147.

Harrison, D.E., Strong, R., Sharp, Z.D., Nelson, J.F., Astle, C.M., Flurkey, K., Nadon, N.L., Wilkinson, J.E., Frenkel, K., Carter, C.S., Pahor, M., Javors, M.A., Fernandez, E., Miller, R.A., 2009. Rapamycin fed late in life extends lifespan in genetically heterogeneous mice. Nature 460, 392-395.

Hars, E.S., Qi, H., Ryazanov, A.G., Jin, S., Cai, L., Hu, C., Liu, L.F., 2007. Autophagy regulates ageing in C. elegans. Autophagy 3, 93-95.

Hashimoto, Y., Ookuma, S., Nishida, E., 2009. Lifespan extension by suppression of autophagy genes in Caenorhabditis elegans. Genes to Cells 14, 717-726.

Hay, N., Sonenberg, N., 2004. Upstream and downstream of mTOR. Genes and Development 18, 1926-1945

He, C., Klionsky, D.J., 2009. Regulation mechanisms and signaling pathways of autophagy. Annual Review of Genetics 43, 67-93.

Heidler, T., Hartwig, K., Daniel, H., Wenzel, U., 2010. Caenorhabditis elegans lifespan extension caused by treatment with an orally active ROS-generator is dependent on DAF-16 and SIR-2.1. Biogerontology 11, 183-195.

Helfand, S.L., Rogina, B., 2003. Genetics of aging in the fruit fly, Drosophila melanogaster. Annual Review of Genetics 37, 329-348.

Heydari, A.R., You, S., Takahashi, R., Gutsmann-Conrad, A., Sarge, K.D., Richardson, A., 2000. Age-related alterations in the activation of heat shock transcription factor 1 in rat hepatocytes. Experimental Cell Research 256, 83-93.

Honda, Y., Honda, S., 1999. The daf- 2 gene network for longevity regulates oxidative stress resistance and Mn-superoxide dismutase gene expression in Caenorhabditis elegans. FASEB Journal 13, 1385-1393.

Honjoh, S., Yamamoto, T., Uno, M., Nishida, E., 2009. Signalling through RHEB1 mediates intermittent fasting-induced longevity in C. elegans. Nature 457 , 726-730.

Houtkooper, R.H., Pirinen, E., Auwerx, J., 2012. Sirtuins as regulators of metabolism and healthspan. Nature Reviews Molecular Cell Biology 13, 225-238.

Hsu, A.L., Murphy, C.T., Kenyon, C., 2003. Regulation of aging and age-related disease by DAF-16 and heat-shock factor. Science 300, 1142-1145.

Huang, W.P., Klionsky, D.J., 2002. Autophagy in yeast: a review of the molecular machinery. Cell Structure and Function 27, 409-420.

Hwang, A.B., Lee, S.J., 2011. Regulation of life span by mitochondrial respiration: the HIF-1 and ROS connection. Aging (Albany, NY) 3, 304-310.

Hwangbo, D.S., Gershman, B., Tu, M.P., Palmer, M., Tatar, M., 2004. Drosophila dFOXO controls lifespan and regulates insulin signalling in brain and fat body. Nature $429,562-566$

Ibanez, P., Lesage, S., Lohmann, E., Thobois, S., De Michele, G., Borg, M., Agid, Y., Durr, A., Brice, A., 2006. Mutational analysis of the PINK1 gene in early-onset parkinsonism in Europe and North Africa. Brain: A Journal of Neurology 129, 686-694.

Irobi, J., Van Impe, K., Seeman, P., Jordanova, A., Dierick, I., Verpoorten, N., Michalik, A., De Vriendt, E., Jacobs, A., Van Gerwen, V., Vennekens, K., Mazanec, R., Tournev, I., Hilton-Jones, D., Talbot, K., Kremensky, I., Van Den Bosch, L., Robberecht, W., Van Vandekerckhove, J., Van Broeckhoven, C., Gettemans, J., De Jonghe, P., Timmerman, V., 2004. Hot-spot residue in small heat-shock protein 22 causes distal motor neuropathy. Nature Genetics 36, 597-601.
Ishii, N., Fujii, M., Hartman, P.S., Tsuda, M., Yasuda, K., Senoo-Matsuda, N., Yanase, S., Ayusawa, D., Suzuki, K., 1998. A mutation in succinate dehydrogenase cytochrome $b$ causes oxidative stress and ageing in nematodes. Nature 394 , 694-697.

Jang, M., Cai, L., Udeani, G.O., Slowing, K.V., Thomas, C.F., Beecher, C.W., Fong, H.H., Farnsworth, N.R., Kinghorn, A.D., Mehta, R.G., Moon, R.C., Pezzuto, J.M., 1997. Cancer chemopreventive activity of resveratrol, a natural product derived from grapes. Science 275, 218-220.

Jia, K., Chen, D., Riddle, D.L., 2004. The TOR pathway interacts with the insulin signaling pathway to regulate C. elegans larval development, metabolism and life span. Development 131, 3897-3906.

Jia, K., Hart, A.C., Levine, B., 2007. Autophagy genes protect against disease caused by polyglutamine expansion proteins in Caenorhabditis elegans. Autophagy 3 , 21-25.

Jia, K., Levine, B., 2007. Autophagy is required for dietary restriction-mediated life span extension in C. elegans. Autophagy 3, 597-599.

Jin, S.M., Youle, R.J., 2012. PINK1- and Parkin-mediated mitophagy at a glance. Journal of Cell Science 125, 795-799.

Johnson, T.E., Henderson, S., Murakami, S., de Castro, E., de Castro, S.H., Cypser, J., Rikke, B., Tedesco, P., Link, C., 2002. Longevity genes in the nematode Caenorhabditis elegans also mediate increased resistance to stress and prevent disease. Journal of Inherited Metabolic Disease 25, 197-206.

Juhasz, G., Erdi, B., Sass, M., Neufeld, T.P., 2007a. Atg7-dependent autophagy promotes neuronal health, stress tolerance, and longevity but is dispensable for metamorphosis in Drosophila. Genes and Development 21, 3061-3066.

Juhasz, G., Puskas, L.G., Komonyi, O., Erdi, B., Maroy, P., Neufeld, T.P., Sass, M., 2007b. Gene expression profiling identifies FKBP39 as an inhibitor of autophagy in larval Drosophila fat body. Cell Death and Differentiation 14, 1181-1190.

Junger, M.A., Rintelen, F., Stocker, H., Wasserman, J.D., Vegh, M., Radimerski, T., Greenberg, M.E., Hafen, E., 2003. The Drosophila forkhead transcription factor FOXO mediates the reduction in cell number associated with reduced insulin signaling. Journal of Biology 2, 20.

Kaeberlein, M., McVey, M., Guarente, L., 1999. The SIR2/3/4 complex and SIR2 alone promote longevity in Saccharomyces cerevisiae by two different mechanisms. Genes and Development 13, 2570-2580.

Kaeberlein, M., Powers 3rd, R.W., Steffen, K.K., Westman, E.A., Hu, D., Dang, N., Kerr, E.O., Kirkland, K.T., Fields, S., Kennedy, B.K., 2005. Regulation of yeast replicative life span by TOR and Sch9 in response to nutrients. Science 310 1193-1196.

Kaeberlein, M., Kennedy, B.K., 2008. Protein translation, 2008. Aging Cell 7, 777-782.

Kaeberlein, T.L., Smith, E.D., Tsuchiya, M., Welton, K.L., Thomas, J.H., Fields, S., Kennedy, B.K., Kaeberlein, M., 2006. Lifespan extension in Caenorhabditis elegans by complete removal of food. Aging Cell 5, 487-494.

Kanfi, Y., Naiman, S., Amir, G., Peshti, V., Zinman, G., Nahum, L., Bar-Joseph, Z., Cohen, H.Y., 2012. The sirtuin SIRT6 regulates lifespan in male mice. Nature $483,218-221$

Kang, C., You, Y.J., Avery, L., 2007. Dual roles of autophagy in the survival of Caenorhabditis elegans during starvation. Genes and Development 21, 2161-2171.

Kang, C., Avery, L., 2008. To be or not to be, the level of autophagy is the question: dual roles of autophagy in the survival response to starvation. Autophagy 4, 82-84.

Kang, C., Avery, L., 2010. Death-associated protein kinase (DAPK) and signal transduction: fine-tuning of autophagy in Caenorhabditis elegans homeostasis. FEBS Journal 277, 66-73.

Kapahi, P., Zid, B.M., Harper, T., Koslover, D., Sapin, V., Benzer, S., 2004. Regulation of lifespan in Drosophila by modulation of genes in the TOR signaling pathway. Current Biology 14, 885-890.

Kayser, E.B., Sedensky, M.M., Morgan, P.G., 2004. The effects of complex I function and oxidative damage on lifespan and anesthetic sensitivity in Caenorhabditis elegans. Mechanisms of Ageing and Development 125, 455-464.

Kenyon, C., Chang, J., Gensch, E., Rudner, A., Tabtiang, R., 1993. A C. elegans mutant that lives twice as long as wild type. Nature 366, 461-464.

Kenyon, C., 2005. The plasticity of aging: insights from long-lived mutants. Cell 120, 449-460.

Kenyon, C.J., 2010. The genetics of ageing. Nature 464, 504-512.

Kimura, K.D., Tissenbaum, H.A., Liu, Y., Ruvkun, G., 1997. daf-2, an insulin receptorlike gene that regulates longevity and diapause in Caenorhabditis elegans. Science 277, 942-946.

Kirkwood, T.B., 2005. Understanding the odd science of aging. Cell 120, 437-447.

Kirkwood, T.B, 2008. A systematic look at an old problem. Nature 451, 644-647.

Klee, C.B., Haiech, J., 1980. Concerted role of calmodulin and calcineurin in calcium regulation. Annals of the New York Academy of Sciences 356, 43-54.

Klionsky, D.J., Cregg, J.M., Dunn Jr., W.A., Emr, S.D., Sakai, Y., Sandoval, I.V., Sibirny, A., Subramani, S., Thumm, M., Veenhuis, M., Ohsumi, Y., 2003. A unified nomenclature for yeast autophagy-related genes. Developmental Cell 5, 539-545.

Klionsky, D.J., 2005. The molecular machinery of autophagy: unanswered questions. Journal of Cell Science 118, 7-18.

Komatsu, M., Waguri, S., Ueno, T., Iwata, J., Murata, S., Tanida, I., Ezaki, J., Mizushima, N., Ohsumi, Y., Uchiyama, Y., Kominami, E., Tanaka, K., Chiba, T., 2005. Impairment of starvation-induced and constitutive autophagy in Atg7-deficient mice. Journal of Cell Biology 169, 425-434

Kouroku, Y., Fujita, E., Tanida, I., Ueno, T., Isoai, A., Kumagai, H., Ogawa, S., Kaufman, R.J., Kominami, E., Momoi, T., 2007. ER stress (PERK/eIF2alpha phosphorylation) mediates the polyglutamine-induced LC3 conversion, an essential step for autophagy formation. Cell Death and Differentiation 14, 230-239. 
Kourtis, N., Tavernarakis, N., 2009. Autophagy and cell death in model organisms. Cell Death and Differentiation 16, 21-30.

Kourtis, N., Tavernarakis, N., 2011. Cellular stress response pathways and ageing: intricate molecular relationships. EMBO Journal 30, 2520-2531.

Kurz, T., Terman, A., Gustafsson, B., Brunk, U.T., 2008. Lysosomes and oxidative stress in aging and apoptosis. Biochimica et Biophysica Acta 1780, 1291-1303.

Kwon, E.S., Narasimhan, S.D., Yen, K., Tissenbaum, H.A., 2010. A new DAF-16 isoform regulates longevity. Nature $466,498-502$.

Lane, D.P., 1992. Cancer. p53, guardian of the genome. Nature 358, 15-16.

Lapierre, L.R., Gelino, S., Melendez, A., Hansen, M., 2011. Autophagy and lipid metabolism coordinately modulate life span in germline-less C. elegans. Current Biology 21, 1507-1514.

Lee, G.D., Wilson, M.A., Zhu, M., Wolkow, C.A., de Cabo, R., Ingram, D.K., Zou, S., 2006. Dietary deprivation extends lifespan in Caenorhabditis elegans. Aging Cell 5, 515-524.

Lee, I.H., Cao, L., Mostoslavsky, R., Lombard, D.B., Liu, J., Bruns, N.E., Tsokos, M., Alt, F.W., Finkel, T., 2008. A role for the NAD-dependent deacetylase Sirt1 in the regulation of autophagy. Proceedings of the National Academy of Sciences of the United States of America 105, 3374-3379.

Lee, S.B., Kim, S., Lee, J., Park, J., Lee, G., Kim, Y., Kim, J.M., Chung, J., 2007. ATG1, an autophagy regulator, inhibits cell growth by negatively regulating $\mathrm{S} 6$ kinase. EMBO Reports 8, 360-365.

Lee, S.J., Hwang, A.B., Kenyon, C., 2010. Inhibition of respiration extends C. elegan life span via reactive oxygen species that increase HIF-1 activity. Current Biology 20, 2131-2136.

Levine, A.J., 1997. p53, the cellular gatekeeper for growth and division. Cell 88, 323-331.

Levine, B., Klionsky, D.J., 2004. Development by self-digestion: molecular mechanisms and biological functions of autophagy. Developmental Cell 6, 463-477.

Levine, B., Kroemer, G., 2008. Autophagy in the pathogenesis of disease. Cell 132 27-42.

Liang, J., Shao, S.H., Xu, Z.X., Hennessy, B., Ding, Z., Larrea, M., Kondo, S., Dumont D.J., Gutterman, J.U., Walker, C.L., Slingerland, J.M., Mills, G.B., 2007. The energy sensing LKB1-AMPK pathway regulates p27(kip1) phosphorylation mediating the decision to enter autophagy or apoptosis. Nature Cell Biology 9, 218-224.

Libina, N., Berman, J.R., Kenyon, C., 2003. Tissue-specific activities of C. elegans DAF16 in the regulation of lifespan. Cell 115, 489-502.

Lin, K., Dorman, J.B., Rodan, A., Kenyon, C., 1997. daf-16: an HNF-3/forkhead family member that can function to double the life-span of Caenorhabditis elegans. Science 278, 1319-1322.

Lin, Y.J., Seroude, L., Benzer, S., 1998. Extended life-span and stress resistance in the Drosophila mutant methuselah. Science 282, 943-946.

Ling, D., Salvaterra, P.M., 2011. Brain aging and Abeta ${ }_{1-42}$ neurotoxicity converge via deterioration in autophagy-lysosomal system: a conditional Drosophila model linking Alzheimer's neurodegeneration with aging. Acta Neuropathologica 121 183-191.

Liu, Q, Berchner-Pfannschmidt, U., Moller, U., Brecht, M., Wotzlaw, C. Acker, $\mathrm{H}$, Jungermann, K., Kietzmann, T., 2004. A Fenton reaction at the endoplasmic reticulum is involved in the redox control of hypoxia-inducible gene expression. Proceedings of the National Academy of Sciences of the United States of America 101, 4302-4307.

Longo, V.D., Kennedy, B.K., 2006. Sirtuins in aging and age-related disease. Cell 126, 257-268.

Luo, X., Puig, O., Hyun, J., Bohmann, D., Jasper, H., 2007. Foxo and Fos regulate the decision between cell death and survival in response to UV irradiation. EMBO Journal 26, 380-390.

Luong, N., Davies, C.R., Wessells, R.J., Graham, S.M., King, M.T., Veech, R., Bodmer, R. Oldham, S.M., 2006. Activated FOXO-mediated insulin resistance is blocked by reduction of TOR activity. Cell Metabolism 4, 133-142.

Madeo, F., Eisenberg, T., Buttner, S., Ruckenstuhl, C., Kroemer, G., 2010. Spermidine: a novel autophagy inducer and longevity elixir. Autophagy 6, 160-162.

Markaki, M., Tavernarakis, N., 2010. Modeling human diseases in Caenorhabditis elegans. Biotechnology Journal 5, 1261-1276.

Martin, D.E., Hall, M.N., 2005. The expanding TOR signaling network. Current Opinion in Cell Biology 17, 158-166.

Martins, I., Galluzzi, L., Kroemer, G., 2011. Hormesis, cell death and aging. Aging (Albany, NY) 3, 821-828.

Mathew, R., Karp, C.M., Beaudoin, B., Vuong, N., Chen, G., Chen, H.Y., Bray, K., Reddy A., Bhanot, G., Gelinas, C., Dipaola, R.S., Karantza-Wadsworth, V., White, E., 2009. Autophagy suppresses tumorigenesis through elimination of p62. Cell 137, 1062-1075.

McPhee, C.K., Baehrecke, E.H., 2009. Autophagy in Drosophila melanogaster. Biochimica et Biophysica Acta 1793, 1452-1460.

Melendez, A., Talloczy, Z., Seaman, M., Eskelinen, E.L., Hall, D.H., Levine, B., 2003. Autophagy genes are essential for dauer development and life-span extension in C. elegans. Science 301, 1387-1391.

Melendez, A., Levine, B., 2009. Autophagy in C. elegans. WormBook 24, 1-26.

Menzies, F.M., Hourez, R., Imarisio, S., Raspe, M., Sadiq, O., Chandraratna, D., O'Kane C., Rock, K.L., Reits, E., Goldberg, A.L., Rubinsztein, D.C., 2010. Puromycinsensitive aminopeptidase protects against aggregation-prone proteins via autophagy. Human Molecular Genetics 19, 4573-4586.

Milton, V.J., Sweeney, S.T., 2012. Oxidative stress in synapse development and function. Developmental Neurobiology 72, 100-110.

Morselli, E., Maiuri, M.C., Markaki, M., Megalou, E., Pasparaki, A., Palikaras, K., Criollo, A., Galluzzi, L., Malik, S.A., Vitale, I., Michaud, M., Madeo, F., Tavernarakis, N.,
Kroemer, G., 2010a. The life span-prolonging effect of sirtuin-1 is mediated by autophagy. Autophagy 6, 186-188.

Morselli, E., Maiuri, M.C., Markaki, M., Megalou, E., Pasparaki, A., Palikaras, K. Criollo, A., Galluzzi, L., Malik, S.A., Vitale, I., Michaud, M., Madeo, F., Tavernarakis, N., Kroemer, G., 2010b. Caloric restriction and resveratrol promote longevity through the Sirtuin-1-dependent induction of autophagy. Cell Death and Disease $1, \mathrm{e} 10$.

Morselli, E., Marino, G., Bennetzen, M.V., Eisenberg, T., Megalou, E., Schroeder S., Cabrera, S., Benit, P., Rustin, P., Criollo, A., Kepp, O., Galluzzi, L., Shen, S., Malik, S.A., Maiuri, M.C., Horio, Y., Lopez-Otin, C., Andersen, J.S., Tavernarakis, N., Madeo, F., Kroemer, G., 2011. Spermidine and resveratrol induce autophagy by distinct pathways converging on the acetylproteome. Journal of Cell Biology 192, 615-629.

Narendra, D.P., Jin, S.M. Tanaka, A. Suen, D.F. Gautier, C.A., Shen, J., Cookson, M.R Youle, R.J., 2010. PINK1 is selectively stabilized on impaired mitochondria to activate Parkin. PLoS Biology 8, e1000298.

Neufeld, T.P., 2010. TOR-dependent control of autophagy: biting the hand that feeds Current Opinion in Cell Biology 22, 157-168.

Nezis, I., 2012. Selective autophagy in Drosophila. International Journal of Cell Biology, http://dx.doi.org/10.1155/2012/146767.

Nojima, H., Tokunaga, C., Eguchi, S., Oshiro, N., Hidayat, S., Yoshino, K., Hara, K. Tanaka, N., Avruch, J., Yonezawa, K., 2003. The mammalian target of rapamycin (mTOR) partner, raptor, binds the mTOR substrates p70 S6 kinase and 4E-BP1 through their TOR signaling (TOS) motif. Journal of Biological Chemistry 278 15461-15464

Ogg, S., Paradis, S., Gottlieb, S., Patterson, G.I., Lee, L., Tissenbaum, H.A., Ruvkun, G., 1997. The Fork head transcription factor DAF-16 transduces insulin-like metabolic and longevity signals in C. elegans. Nature 389, 994-999.

Oh, S.W., Mukhopadhyay, A., Svrzikapa, N., Jiang, F., Davis, R.J., Tissenbaum, H.A 2005. JNK regulates lifespan in Caenorhabditis elegans by modulating nuclear translocation of forkhead transcription factor/DAF-16. Proceedings of the National Academy of Sciences of the United States of America 102, 4494-4499.

Pallanck, L., Greenamyre, J.T., 2006. Neurodegenerative disease: pink, parkin and the brain. Nature 441, 1058.

Pan, K.Z., Palter, J.E., Rogers, A.N., Olsen, A., Chen, D., Lithgow, G.J., Kapahi, P., 2007. Inhibition of mRNA translation extends lifespan in Caenorhabditis elegans. Aging Cell 6, 111-119.

Pandey, U.B., Nie, Z., Batlevi, Y., McCray, B.A., Ritson, G.P., Nedelsky, N.B., Schwartz S.L., DiProspero, N.A., Knight, M.A., Schuldiner, O., Padmanabhan, R., Hild, M. Berry, D.L., Garza, D., Hubbert, C.C., Yao, T.P., Baehrecke, E.H., Taylor, J.P., 2007. HDAC6 rescues neurodegeneration and provides an essential link between autophagy and the UPS. Nature 447, 859-863.

Panowski, S.H., Wolff, S., Aguilaniu, H., Durieux, J., Dillin, A., 2007. PHA-4/Foxa mediates diet-restriction-induced longevity of C. elegans. Nature 447, 550-555.

Parsons, P.A., 1995. Inherited stress resistance and longevity: a stress theory of ageing. Heredity 75 (Pt 2), 216-221.

Pattingre, S., Tassa, A., Ou, X., Garuti, R., Liang, X.H., Mizushima, N., Packer, M. Schneider, M.D., Levine, B., 2005. Bcl-2 antiapoptotic proteins inhibit Beclin 1-dependent autophagy. Cell 122, 927-939.

Poole, A.C., Thomas, R.E., Andrews, L.A., McBride, H.M., Whitworth, A.J., Pallanck, L.J., 2008. The PINK1/Parkin pathway regulates mitochondrial morphology. Proceedings of the National Academy of Sciences of the United States of America $105,1638-1643$.

Poole, A.C., Thomas, R.E., Yu, S., Vincow, E.S., Pallanck, L., 2010. The mitochondrial fusion-promoting factor mitofusin is a substrate of the PINK1/parkin pathway. PLoS One 5, e10054.

Py, B.F., Boyce, M., Yuan, J., 2009. A critical role of eEF-2K in mediating autophagy in response to multiple cellular stresses. Autophagy 5, 393-396.

Rabinowitz, J.D., White, E., 2010. Autophagy and metabolism. Science 330 1344-1348.

Ravikumar, B., Vacher, C. Berger, Z Davies, JE, Luo, S., Oroz, L. G Scaravilli, F. Easton, D.F., Duden, R., O'Kane, C.J., Rubinsztein, D.C., 2004. Inhibition of mTOR induces autophagy and reduces toxicity of polyglutamine expansions in fly and mouse models of Huntington disease. Nature Genetics 36, 585-595.

Ravikumar, B., Acevedo-Arozena, A., Imarisio, S., Berger, Z., Vacher, C., O’Kane, C.J., Brown, S.D., Rubinsztein, D.C., 2005. Dynein mutations impair autophagic clearance of aggregate-prone proteins. Nature Genetics 37, 771-776.

Rea, S.L., 2005. Metabolism in the Caenorhabditis elegans Mit mutants. Experimental Gerontology 40, 841-849.

Reinke, S.N., Hu, X., Sykes, B.D., Lemire, B.D., 2010. Caenorhabditis elegans diet significantly affects metabolic profile, mitochondrial DNA levels, lifespan and brood size. Molecular Genetics and Metabolism 100, 274-282.

Ren, C., Finkel, S.E., Tower, J., 2009. Conditional inhibition of autophagy genes in adult Drosophila impairs immunity without compromising longevity. Experimenta Gerontology 44, 228-235.

Riezman, H., 2004. Why do cells require heat shock proteins to survive heat stress? Cell Cycle 3, 61-63.

Rogina, B., Helfand, S.L., Frankel, S., 2002. Longevity regulation by Drosophila Rpd3 deacetylase and caloric restriction. Science 298, 1745.

Rogina, B., Helfand, S.L., 2004. Sir2 mediates longevity in the fly through a pathway related to calorie restriction. Proceedings of the National Academy of Sciences of the United States of America 101, 15998-16003.

Rubinsztein, D.C., Ravikumar, B., Acevedo-Arozena, A., Imarisio, S., O’Kane, C.J., Brown, S.D., 2005. Dyneins, autophagy, aggregation and neurodegeneration. Autophagy 1, 177-178. 
Rubinsztein, D.C., Marino, G., Kroemer, G, 2011. Autophagy and aging. Cell 146, 682-695.

Rutkowski, R., Hofmann, K., Gartner, A., 2011. Phylogeny and function of the invertebrate 553 superfamily. Cold Spring Harbor Perspectives in Biology 2, a001131.

Salih, D.A. Brunet, A, 2008. FoxO transcription factors in the maintenance of cellular homeostasis during aging. Current Opinion in Cell Biology 20,126-136.

Samara, C., Syntichaki, P., Tavernarakis, N., 2008. Autophagy is required for necrotic cell death in Caenorhabditis elegans. Cell Death and Differentiation 15, 105-112.

Samara, C., Tavernarakis, N., 2008. Autophagy and cell death in Caenorhabditis elegans. Current Pharmaceutical Design 14, 97-115.

Samokhvalov, V., Scott, B.A., Crowder, C.M., 2008. Autophagy protects against hypoxic injury in C. elegans. Autophagy 4, 1034-1041

Sarbassov, D.D., Ali, S.M., Sabatini, D.M., 2005. Growing roles for the mTOR pathway. Current Opinion in Cell Biology 17, 596-603.

Sarkar, S., Perlstein, E.O., Imarisio, S., Pineau, S., Cordenier, A., Maglathlin, R.L., Webster, J.A., Lewis, T.A., O’Kane, C.J., Schreiber, S.L., Rubinsztein, D.C., 2007. Small molecules enhance autophagy and reduce toxicity in Huntington's disease models. Nature Chemical Biology 3, 331-338.

Scherz-Shouval, R., Shvets, E., Fass, E., Shorer, H., Gil, L., Elazar, Z., 2007. Reactive oxygen species are essential for autophagy and specifically regulate the activity of Atg4. EMBO Journal 26, 1749-1760.

Schulz, T.J., Zarse, K., Voigt, A., Urban, N., Birringer, M., Ristow, M., 2007. Glucose restriction extends Caenorhabditis elegans life span by inducing mitochondrial respiration and increasing oxidative stress. Cell Metabolism 6, 280-293.

Scott, R.C., Schuldiner, O., Neufeld, T.P., 2004. Role and regulation of starvationinduced autophagy in the Drosophila fat body. Developmental Cell 7, 167-178.

Senoo-Matsuda, N., Hartman, P.S., Akatsuka, A., Yoshimura, S., Ishii, N., 2003. A complex II defect affects mitochondrial structure, leading to ced-3- and ced-4-dependent apoptosis and aging. Journal of Biological Chemistry 278 22031-22036

Shaw, W.M., Luo, S., Landis, J., Ashraf, J., Murphy, C.T., 2007. The C. elegans TGFbeta dauer pathway regulates longevity via insulin signaling. Current Biology $17,1635-1645$.

Sheaffer, K.L., Updike, D.L., Mango, S.E., 2008. The Target of Rapamycin pathway antagonizes pha-4/FoxA to control development and aging. Current Biology 18, 1355-1364.

Shen, W., Ganetzky, B., 2009. Autophagy promotes synapse development in Drosophila. Journal of Cell Biology 187, 71-79.

Simonsen, A., Cumming, R.C., Brech, A., Isakson, P., Schubert, D.R., Finley, K.D., 2008. Promoting basal levels of autophagy in the nervous system enhances longevity and oxidant resistance in adult Drosophila. Autophagy 4, 176-184.

Soukas, A.A., Kane, E.A., Carr, C.E., Melo, J.A., Ruvkun, G., 2009. Rictor/TORC2 regulates fat metabolism, feeding, growth, and life span in Caenorhabditis elegans. Genes and Development 23, 496-511.

Stetler, R.A., Gan, Y., Zhang, W., Liou, A.K., Gao, Y., Cao, G., Chen, J., 2010. Heat shock proteins: cellular and molecular mechanisms in the central nervous system. Progress in Neurobiology 92, 184-211.

Sun, J., Tower, J., 1999. FLP recombinase-mediated induction of $\mathrm{Cu} / \mathrm{Zn}$-superoxide dismutase transgene expression can extend the life span of adult Drosophila melanogaster flies. Molecular and Cellular Biology 19, 216-228.

Sun, J., Folk, D., Bradley, T.J., Tower, J., 2002. Induced overexpression of mitochondrial Mn-superoxide dismutase extends the life span of adult Drosophila melanogaster. Genetics 161, 661-672.

Syntichaki, P., Troulinaki, K., Tavernarakis, N., 2007. eIF4E function in somatic cells modulates ageing in Caenorhabditis elegans. Nature 445, 922-926.

Talloczy, Z. Jiang, W., Virgin 4th., H.W., Leib, D.A., Scheuner, D., Kaufman, R.J., Eskelinen, E.L., Levine, B., 2002. Regulation of starvation- and virus-induced autophagy by the eIF2alpha kinase signaling pathway. Proceedings of the National Academy of Sciences of the United States of America 99, 190-195.

Tanaka, A., 2010. Parkin-mediated selective mitochondrial autophagy, mitophagy: Parkin purges damaged organelles from the vital mitochondrial network. FEBS Letters 584, 1386-1392.

Tang, B.S., Zhao, G.H., Luo, W., Xia, K., Cai, F., Pan, Q., Zhang, R.X., Zhang, F.F., Liu, X.M., Chen, B., Zhang, C., Shen, L., Jiang, H., Long, Z.G., Dai, H.P., 2005. Small heat-shock protein 22 mutated in autosomal dominant Charcot-Marie-Tooth disease type 2L. Human Genetics 116, 222-224.

Tasdemir, E., Maiuri, M.C., Galluzzi, L., Vitale, I., Djavaheri-Mergny, M., D’Amelio, M., Criollo, A., Morselli, E., Zhu, C., Harper, F., Nannmark, U., Samara, C., Pinton, P., Vicencio, J.M., Carnuccio, R., Moll, U.M., Madeo, F., Paterlini-Brechot, P., Rizzuto, R., Szabadkai, G., Pierron, G., Blomgren, K., Tavernarakis, N., Codogno, P., Cecconi, F., Kroemer, G., 2008. Regulation of autophagy by cytoplasmic p53. Nature Cell Biology 10, 676-687.

Tatar, M., Kopelman, A., Epstein, D., Tu, M.P., Yin, C.M., Garofalo, R.S., 2001. A mutant Drosophila insulin receptor homolog that extends life-span and impairs neuroendocrine function. Science 292, 107-110.

Tavernarakis, N., Pasparaki, A., Tasdemir, E., Maiuri, M.C., Kroemer, G., 2008. The effects of p53 on whole organism longevity are mediated by autophagy. Autophagy 4, 870-873.

Tee, A.R., Blenis, J., 2005. mTOR, translational control and human disease. Seminars in Cell and Developmental Biology 16, 29-37.

Tian, E., Wang, F., Han, J., Zhang, H., 2009. epg-1 functions in autophagy-regulated processes and may encode a highly divergent Atg13 homolog in C. elegans. Autophagy 5, 608-615.

Tian, Y., Li, Z., Hu, W., Ren, H., Tian, E., Zhao, Y., Lu, Q., Huang, X., Yang, P., Li, X., Wang, X., Kovacs, A.L., Yu, L., Zhang, H., 2010. C. elegans screen identifies autophagy genes specific to multicellular organisms. Cell 141, 1042-1055.
Tissenbaum, H.A., Guarente, L., 2001. Increased dosage of a sir-2 gene extends lifespan in Caenorhabditis elegans. Nature 410, 227-230.

Toivonen, J.M., Walker, G.A., Martinez-Diaz, P., Bjedov, I., Driege, Y., Jacobs, H.T., Gems, D., Partridge, L., 2007. No influence of Indy on lifespan in Drosophila after correction for genetic and cytoplasmic background effects. PLoS Genetics 3, e95.

Toth, M.L., Simon, P., Kovacs, A.L., Vellai, T., 2007. Influence of autophagy genes on ion-channel-dependent neuronal degeneration in Caenorhabditis elegans. Journal of Cell Science 120, 1134-1141.

Toth, M.L., Sigmond, T., Borsos, E., Barna, J., Erdelyi, P., Takacs-Vellai, K., Orosz, L. Kovacs, A.L., Csikos, G., Sass, M., Vellai, T., 2008. Longevity pathways converge on autophagy genes to regulate life span in Caenorhabditis elegans. Autophagy $4,330-338$.

Tower, J., 2011. Heat shock proteins and Drosophila aging. Experimental Gerontology 46, 355-362.

Tullet, J.M., Hertweck, M., An, J.H., Baker, J., Hwang, J.Y., Liu, S., Oliveira, R.P., Baumeister, R., Blackwell, T.K., 2008. Direct inhibition of the longevity-promoting factor SKN-1 by insulin-like signaling in C. elegans. Cell 132, 1025-1038.

Twig, G., Elorza, A., Molina, A.J., Mohamed, H., Wikstrom, J.D., Walzer, G., Stiles, L., Haigh, S.E., Katz, S., Las, G., Alroy, J., Wu, M., Py, B.F., Yuan, J., Deeney, J.T., Corkey, B.E., Shirihai, O.S., 2008. Fission and selective fusion govern mitochondrial segregation and elimination by autophagy. EMBO Journal 27, 433-446.

Valente, E.M., Abou-Sleiman, P.M., Caputo, V., Muqit, M.M., Harvey, K., Gispert, S., Ali, Z., Del Turco, D., Bentivoglio, A.R., Healy, D.G., Albanese, A., Nussbaum, R., Gonzalez-Maldonado, R., Deller, T., Salvi, S., Cortelli, P., Gilks, W.P., Latchman, D.S., Harvey, R.J., Dallapiccola, B., Auburger, G., Wood, N.W., 2004. Hereditary early-onset Parkinson's disease caused by mutations in PINK1. Science 304, 1158-1160.

Vellai, T., Takacs-Vellai, K., Zhang, Y., Kovacs, A.L., Orosz, L., Muller, F., 2003. Genetics: influence of TOR kinase on lifespan in C. elegans. Nature 426, 620.

Vellai, T., Takacs-Vellai, K., Sass, M., Klionsky, D.J., 2009. The regulation of aging: does autophagy underlie longevity? Trends in Cell Biology 19, 487-494.

Ventura, N., Rea, S.L., Testi, R., 2006. Long-lived C. elegans mitochondrial mutants as a model for human mitochondrial-associated diseases. Experimental Gerontology 41, 974-991.

Ventura, N., Rea, S.L., Schiavi, A., Torgovnick, A., Testi, R., Johnson, T.E., 2009. p53/CEP-1 increases or decreases lifespan, depending on level of mitochondrial bioenergetic stress. Aging Cell 8, 380-393.

Viswanathan, M., Kim, S.K., Berdichevsky, A., Guarente, L., 2005. A role for SIR2.1 regulation of ER stress response genes in determining C. elegans life span. Developmental Cell 9, 605-615.

Vos, M.J., Zijlstra, M.P., Kanon, B., van Waarde-Verhagen, M.A., Brunt, E.R., Oosterveld-Hut, H.M., Carra, S., Sibon, O.C., Kampinga, H.H., 2010. HSPB7 is the most potent polyQ aggregation suppressor within the HSPB family of molecular chaperones. Human Molecular Genetics 19, 4677-4693.

Vos, M.J., Zijlstra, M.P., Carra, S., Sibon, O.C., Kampinga, H.H., 2011. Small heat shock proteins, protein degradation and protein aggregation diseases. Autophagy 7 , 101-103.

Vousden, K.H., Lane, D.P., 2007. p53 in health and disease. Nature Reviews Molecular Cell Biology 8, 275-283.

Wang, M.C., Bohmann, D., Jasper, H., 2003. JNK signaling confers tolerance to oxidative stress and extends lifespan in Drosophila. Developmental Cell 5, 811-816.

Wang, M.C., Bohmann, D., Jasper, H., 2005. JNK extends life span and limits growth by antagonizing cellular and organism-wide responses to insulin signaling. Cell $121,115-125$.

Wang, M.C., O’Rourke, E.J., Ruvkun, G., 2008. Fat metabolism links germline stem cells and longevity in C. elegans. Science 322, 957-960.

Wang, Y., Tissenbaum, H.A., 2006. Overlapping and distinct functions for a Caenorhabditis elegans SIR2 and DAF-16/FOXO. Mechanisms of Ageing and Development 127, 48-56.

Winslow, A.R., Chen, C.W., Corrochano, S., Acevedo-Arozena, A., Gordon, D.E., Peden, A.A., Lichtenberg, M., Menzies, F.M., Ravikumar, B., Imarisio, S., Brown, S., O’Kane, C.J., Rubinsztein, D.C., 2010. alpha-Synuclein impairs macroautophagy: implications for Parkinson's disease. Journal of Cell Biology 190, 1023-1037.

Wood, J.G., Rogina, B., Lavu, S., Howitz, K., Helfand, S.L., Tatar, M., Sinclair, D., 2004. Sirtuin activators mimic caloric restriction and delay ageing in metazoans. Nature 430, 686-689.

Wu, H., Wang, M.C., Bohmann, D., 2009. JNK protects Drosophila from oxidative stress by trancriptionally activating autophagy. Mechanisms of Development 126, 624-637.

Wullschleger, S., Loewith, R., Hall, M.N., 2006. TOR signaling in growth and metabolism. Cell 124, 471-484

Xie, Z., Klionsky, D.J., 2007. Autophagosome formation: core machinery and adaptations. Nature Cell Biology 9, 1102-1109.

Yamada, Y., Davis, K.D., Coffman, C.R., 2008. Programmed cell death of primordial germ cells in Drosophila is regulated by p53 and the outsiders monocarboxylate transporter. Development 135, 207-216.

Yamamoto, R., Tatar, M., 2011. Insulin receptor substrate chico acts with the transcription factor FOXO to extend Drosophila lifespan. Aging Cell 10, 729-732.

Yang, W., Hekimi, S., 2010a. Two modes of mitochondrial dysfunction lead independently to lifespan extension in Caenorhabditis elegans. Aging Cell 9, 433-447.

Yang, W., Hekimi, S., 2010b. A mitochondrial superoxide signal triggers increased longevity in Caenorhabditis elegans. PLoS Biology 8, e1000556.

Yang, Y., Ouyang, Y., Yang, L., Beal, M.F., McQuibban, A., Vogel, H., Lu, B., 2008. Pink1 regulates mitochondrial dynamics through interaction with the fission/fusion machinery. Proceedings of the National Academy of Sciences of the United States of America 105, 7070-7075. 
Yang, Z., Klionsky, D.J., 2010. Eaten alive: a history of macroautophagy. Nature Cell Biology 12, 814-822

Ye, K., Ji, C.B., Lu, X.W., Ni, Y.H., Gao, C.L., Chen, X.H., Zhao, Y.P., Gu, G.X., Guo X.R., 2010. Resveratrol attenuates radiation damage in Caenorhabditis elegans by preventing oxidative stress. Journal of Radiation Research 51, 473-479.

Yiu, C.Y., Chen, S.Y., Chang, L.K., Chiu, Y.F., Lin, T.P., 2010. Inhibitory effects of resveratrol on the Epstein-Barr virus lytic cycle. Molecules 15, 7115-7124.

Youle, R.J., Narendra, D.P., 2011. Mechanisms of mitophagy. Nature Reviews Molecular Cell Biology 12, 9-14.
Zhang, F., Liu, J., Shi, J.S., 2010. Anti-inflammatory activities of resveratrol in the brain: role of resveratrol in microglial activation. European Journal of Pharmacology 636, 1-7.

Ziviani, E., Tao, R.N., Whitworth, A.J., 2010. Drosophila parkin requires PINK1 for mitochondrial translocation and ubiquitinates mitofusin. Proceedings of the National Academy of Sciences of the United States of America 107, 5018-5023. 